\title{
Analytic approach to non-slow-roll inflation
}

\author{
Gianmassimo Tasinato \\ Department of Physics, Swansea University, Swansea SA2 8PP, United Kingdom
}

(Received 23 September 2020; accepted 7 January 2021; published 26 January 2021)

\begin{abstract}
In order to produce primordial black holes, or to enhance the spectrum of primordial gravitational waves at interferometer scales, cosmological inflation should include brief periods of nonattractor evolution, with departures from slow-roll conditions. To better understand these systems we develop a model independent, analytic approach to study cosmological fluctuations in single field inflationary models with short periods of slow-roll violation. We Taylor expand the equations for fluctuations in a quantity parametrizing the duration of slow-roll violation, and find solutions at leading order in this quantity. The resulting spectrum of perturbations is described by few parameters, and presents a pronounced dip followed by a rapid growth in amplitude, as typically found in numerical as well as analytical studies of similar scenarios. We determine new analytic relations between the position and depth of the dip, and the maximal enhancement of the spectrum toward small scales. For a single epoch of slow-roll violation we confirm previous studies finding that the steepest slope of the spectrum has spectral index $n_{s}-1=4$. On the other hand, when considering multiple phases of slow-roll violation, the slope of the spectrum is generally enhanced. When two epochs of slow-roll violation occur, the spectral index can reach the value $n_{s}-1=8$, indicating that the slope of the spectrum has memory of the history of non-slow-roll phases during inflation.
\end{abstract}

DOI: 10.1103/PhysRevD.103.023535

\section{INTRODUCTION}

The predictions of single-field slow-roll inflation with respect to the statistics of cosmological fluctuations are in perfect agreement with current cosmological microwave background (CMB) and large scale structure observations. By now, they constitute standard textbook material, see, e.g., [1]. On the other hand, large scale cosmological observations only test a fraction of the total number of e-folds of inflation needed to solve the basic problems of standard big bang cosmology. It is interesting to explore the possibility that during inflation short periods of non-slowroll evolution occur, that leave distinct imprints in the small scale regions of the spectrum of scalar and tensor fluctuations. A strong amplification of the spectrum of scalar curvature fluctuations could overcome the threshold of formation for primordial black holes within single field inflation [2-6] (see [7,8] for reviews). Enhancing the primordial gravitational wave spectrum toward large frequencies would make it detectable with gravitational wave experiments (see, e.g., [9] for a recent study focused on LISA).

Published by the American Physical Society under the terms of the Creative Commons Attribution 4.0 International license. Further distribution of this work must maintain attribution to the author(s) and the published article's title, journal citation, and DOI. Funded by SCOAP ${ }^{3}$.
In this work we develop an analytical, model independent method for studying the spectrum of cosmological fluctuations in single-field inflationary scenarios that include brief, transient departures from slow-roll evolution. Calling $\Delta \tau / \tau_{0}$ a dimensionless quantity parametrizing the duration of the non-slow-roll epoch versus a reference timescale, we organize the evolution equations of fluctuations in a Taylor series in this quantity, and we are able to solve the equations at first order in $\Delta \tau / \tau_{0}$. We derive the properties of the corresponding spectrum of fluctuations in scenarios where its size is much enhanced toward small scales. The spectrum is described by few parameters, and presents a pronounced dip followed by a rapid growth in amplitude, as typically found in numerical as well as analytical studies of non-slow-roll scenarios. The simplicity of our results suggest new quantitative relations between the features of the spectrum. For example, we relate the position and depth of the dip with the maximal enhancement of the spectrum toward small scales. For a single epoch of slow-roll violation we confirm previous studies [10-12] finding that the steepest slope of the spectrum has spectral index $n_{s}-1=4$. When considering multiple phases of slow-roll violation, however, the slope of the spectrum can be enhanced. This indicates that the slope of the spectrum has memory of the history of non-slow-roll phases during inflation.

Our work is organized as follows:

(i) In Sec. II we develop our analytical method, which is based on a gradient expansion of the perturbation 
equations and on a Taylor series on the duration $\Delta \tau$ of the non-slow-roll era. After introductory sections with motivations (Secs. II A and II B) we show that the system of equations can be analytically solved at first order in a $\Delta \tau$ expansion, leading to a solution of the mode function that depends on few parameters (Secs. II C-II E). Section II F briefly compares our approach with other analytical methods discussed in the literature.

(ii) In Sec. III we apply our analytic results to compute the power spectrum of fluctuations and the corresponding spectral index in models of single field inflation containing phases of non-slow-roll evolution. In Secs. III A 1, III A 2 we focus on scenarios containing a single non-slow-roll era: we determine new analytic formulas characterizing the properties of the spectrum, and confirm previous results on slope of the spectral index. Section III B instead considers for the first time scenarios with multiple non-slow-roll epochs, nested between phases of quasi-de Sitter expansion. We find that the slope of the spectrum in small scale regions toward the peak can be much steeper than in the previous case, and we discuss the physical implications of our results.

(iii) Section IV contains our conclusions, followed by Appendix A presenting an explicit model of nonslow-roll inflation where our formulas can be applied, and by Appendix B comparing the results of our method with alternative analytical and numerical approaches to the problem.

\section{ANALYTIC FORMULAS FOR THE MODE FUNCTION OF FLUCTUATIONS DURING TRANSIENT EPOCHS OF SLOW-ROLL VIOLATION}

\section{A. The standard case}

We focus on an inflationary epoch of cosmological expansion in single-field inflation, during which for most of the process the metric is well approximated by pure de Sitter space

$d s^{2}=a^{2}(\tau)\left(-d \tau^{2}+d \vec{x}^{2}\right), \quad a(\tau)=-\frac{1}{H_{0} \tau}, \quad \tau \leq 0$,

up to subleading slow-roll corrections, that we neglect in this work. Here, $\tau$ is the conformal time and $H_{0}$ the (nearly) constant Hubble parameter during inflation. We consider transient departures from the previous metric, and we are interested in studying the statistical distribution of the massless fluctuations of fields propagating in this spacetime. Such fluctuations are controlled by the following quadratic action in Fourier space:

$$
S=\frac{1}{2} \int d \tau d^{3} k z^{2}(\tau)\left[\psi_{k}^{\prime 2}(\tau)+k^{2} \psi_{k}^{2}(\tau)\right] .
$$

The function $z(\tau)$ is dubbed pump field: it is model dependent, and characterizes the dynamics of the fluctuations under study. The structure of the previous quadratic action is generic in single field inflation. It can describe the dynamics of the Mukhanov-Sasaki variable $\zeta_{k}$ controlling curvature fluctuations in single-field vanilla models of inflation, where $z \propto a \dot{\phi} / H_{0}$ with $\phi$ the inflaton field; or the dynamics of massless spin-2 (tensor) or spin-0 (scalar) fluctuations around pure de Sitter, and then $z \propto a$ (see, e.g., [1]). But more general possibilities can occur, which are interesting to investigate. In a regime of slow-roll singlefield inflation, the pump field has the generic profile

$$
z_{\mathrm{dS}}(\tau)=-\frac{c_{0}}{H_{0} \tau}
$$

up to small subleading slow-roll corrections, which we neglect in this work. Here, $c_{0}$ denotes a (nearly-)constant quantity depending on the system one considers. Working with a pump field as in Eq. (2.3) it is straightforward to solve exactly the evolution equation for the mode $\psi_{k}(\tau)$ in this pure de Sitter limit of slow-roll inflation. We get

$\psi_{k}(\tau)=-\frac{i}{\sqrt{2 k^{3}}} \frac{H_{0}}{c_{0}}\left[\mathcal{C}_{1}(1+i k \tau) e^{-i k \tau}+\mathcal{C}_{2}(1-i k \tau) e^{i k \tau}\right]$,

for two arbitrary complex constants $\mathcal{C}_{1,2}$, independent from time. The solution that matches the Bunch-Davies vacuum at early times selects $\mathcal{C}_{1}=1, \mathcal{C}_{2}=0$ and reads (see, e.g., [1])

$$
\psi_{k}(\tau)=-i \frac{e^{-i k \tau}}{\sqrt{2 k^{3}}} \frac{H_{0}}{c_{0}}(1+i k \tau) .
$$

\section{B. Transient deviations from slow-roll evolution: our motivations and our approach}

We investigate general properties of the spectrum of fluctuations $\psi_{k}(\tau)$ in scenarios where there exist brief, transient epochs of violation of slow-roll conditions. During the brief non-slow-roll epochs the pump field $z(\tau)$ is not described by the simple profile of Eq. (2.3). In these phases we do not expect a regime of attractor inflation, hence the would-be decaying mode becomes temporarily important, affecting the features of the power spectrum.

One motivation for considering this possibility is to amplify the spectrum of scalar curvature fluctuations at small scales, so to overcome the threshold of formation for primordial black holes within single field inflation [2-6] (see $[7,8]$ for reviews). Another motivation is to investigate models of inflation that amplify the amplitude primordial tensor at frequencies directly testable with gravitational wave experiments (see, e.g., [9] for a study focused on LISA). Finally, possible anomalous features in the spectra 
of CMB temperature fluctuations and spectral distortions could be explained by a temporary growth of some of the slow-roll parameters, see, e.g., [13] for a detailed review.

So far, various models achieve these aims by designing a short phase of inflationary nonattractor evolution. In one class of models, the first slow-roll parameter $\epsilon_{1}=-\dot{H} / H^{2}$ remains small, while the absolute value of the second slow-roll parameter $\epsilon_{2}=\dot{\epsilon}_{1} /\left(H \epsilon_{1}\right)$ is large, see, e.g., [2,6,14-18]. Nonattractor inflation is typically obtained when the pump field entering in Eq. (2.2) decreases with time, instead of increasing as what happens in the standard case (see Eq. (2.3), and recall that time runs over negative values). A well-studied example of nonattractor evolution is ultra-slow-roll inflation, with $\epsilon_{2}=-6$ and $\epsilon_{1} \sim 0$, and pump field $z(\tau) \propto \tau^{2}$. Such scenario can be well realized in single-field scenarios with inflection points in the inflationary potential (see, e.g., [14,19-22]), where $V_{, \phi} \simeq 0, \epsilon_{1}$ very small, hence

$$
\frac{\ddot{\phi}}{H \dot{\phi}}=-\epsilon_{1}+\frac{\epsilon_{2}}{2} \simeq-3 \Rightarrow \epsilon_{2} \simeq-6 .
$$

The corresponding statistics of fluctuations is related to the standard slow-roll case by a duality [23] which leads to simple, physically transparent formulas for the spectrum of fluctuations (this approach initially developed for the scalar sector of curvature fluctuations can be extended to the tensor sector as well $[24,25])$.

Another class of models include scenarios of "punctuated" inflation, where brief interruptions of quasi-de Sitter expansion lead to temporary enhancements also of the slow-roll parameter $\epsilon_{1}$, that then returns to small values for a final phase of quasi-de Sitter evolution (see, e.g., [26-30] for early works and the review [13]). These setups can be realized in systems whose potentials have steps, or discontinuities in their derivatives (see, e.g., the early papers [31,32]). In this case, analytic approaches are not always available, but numerical methods can be used (see, e.g., [33]).

In this work, instead of studying specific models, we enquire whether there are common features in the properties of the power spectrum of fluctuations as a function of the scale, in scenarios that include brief and transient phases of non-slow-roll evolution. ${ }^{1}$ For this aim, we do not use arguments based on slow-roll expansions, nor need to specify explicit values for slow-roll parameters. Instead, we directly work in terms of the pump field $z(\tau)$. We develop an analytical method to solve the mode equations for the perturbation $\psi_{k}(\tau)$ in the limit of short duration $\Delta \tau$ of nonslow-roll epoch (in comparison with the total duration of inflation), that can be applied to general profiles for the pump field, regardless of the physical origin of the model.

\footnotetext{
${ }^{1}$ The condition of brief nonattractor epoch is needed for avoiding issues with quantum diffusion, see, e.g., [34-36].
}

In the limit of small $\Delta \tau$, we perturbatively Taylor expand the functions involved in this small parameter controlling the duration of nonattractor epoch.

We concretely develop this plan in what follows. First, we fix some notation. We parametrize the pump field as depending on a function $\omega(\tau)$ (the square root is included to simplify the formulas we meet in the next sections)

$$
z(\tau)= \begin{cases}-\sqrt{\omega\left(\tau_{1}\right)} \frac{c_{0}}{H_{0} \tau} & \text { for } \tau<\tau_{1} \\ -\sqrt{\omega(\tau)} \frac{c_{0}}{H_{0} \tau} & \text { for } \tau_{1} \leq \tau \leq \tau_{2} \\ -\sqrt{\omega\left(\tau_{2}\right)} \frac{c_{0}}{H_{0} \tau} & \text { for } \tau>\tau_{2}\end{cases}
$$

Without losing in generality, we choose $\omega\left(\tau_{1}\right)=1$. Nonslow-roll evolution occurs in the time interval $\tau_{1} \leq \tau \leq \tau_{2}$. We denote such interval as

$$
\Delta \tau \equiv \tau_{2}-\tau_{1}
$$

The pump field $z(\tau)$ is a continuous function of time, but its first derivatives can be discontinuous within the interval $\Delta \tau$. In the ultra-slow-roll example discussed above, $z(\tau) \propto$ $\tau^{2}$ hence $\omega(\tau) \propto \tau^{6}$, while in the case of slow-roll inflation $\omega(\tau)$ is nearly constant. More generally, in Fig. 1 we pictorially represent arbitrary profiles for the function $\omega(\tau)$ during the nonattractor evolution.

The conformal time $\tau_{1}$ denotes the conformal time when the inflationary system first leave the slow-roll phase, to enter into the non-slow-roll era. We consider cases in which the duration of non-slow-roll evolution is much shorter than the typical timescales of the systems, that we take of order $\left|\tau_{1}\right|$. This assumption is quantified by the inequality ${ }^{2}$

$$
\sigma \equiv \frac{\Delta \tau}{\left|\tau_{1}\right|} \ll 1
$$

which defines a small parameter $\sigma$, useful for our arguments based on a perturbative expansion.

While in Eq. (2.7) we consider a single phase of nonslow-roll expansion, it is also possible to consider situations with multiple non-slow-roll epochs, and with intermediate phases of slow-roll expansion in between. We postpone these considerations in Sec. III B. In what comes next we elaborate an appropriate Ansatz for the mode function $\psi_{k}$, which allows us to solve the corresponding evolution

\footnotetext{
${ }^{2}$ We prefer to quantify the short duration of the non-slowroll era in terms of the combination $\Delta \tau /\left|\tau_{1}\right|$, instead of the corresponding number of e-folds of inflation. This since in general beyond slow-roll we do not have information about the scale factor $a(\tau)$, and the e-fold number $\Delta N$, during the time interval $\Delta \tau$.
} 

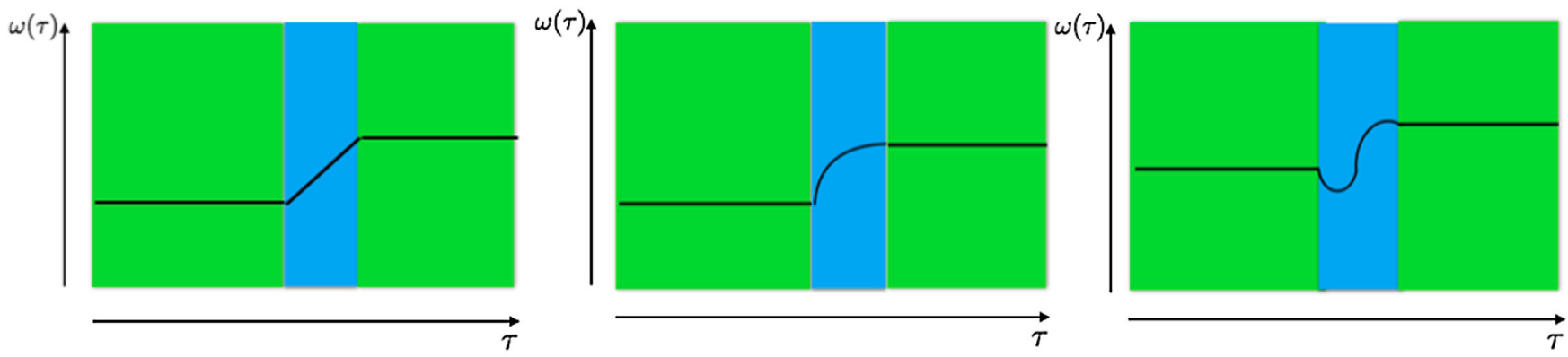

FIG. 1. Pictorial representation of arbitrary profiles for the function $\omega$ in Eq. (2.7) which can give rise to brief periods of slow-roll violation (in blue in the figure).

equations in the limit of short duration (2.9) of non-slowroll evolution.

\section{An Ansatz to describe the non-slow-roll evolution}

In order to study the system, as is customary we rescale

$$
\psi_{k}(\tau) \equiv \frac{\varphi_{k}(\tau)}{z(\tau)} .
$$

The field equation for $\varphi_{k}(\tau)$ reads

$$
\varphi_{k}^{\prime \prime}+\left(k^{2}-\frac{z^{\prime \prime}}{z}\right) \varphi_{k}=0
$$

where we assume a profile for the pump field $z(\tau)$ as in Eq. (2.7).

It is not immediately clear how to design a method to "separate" the dependence from time $\tau$ and scale $k$ in Eq. (2.10), and Taylor expand in small time intervals $\Delta \tau$. We proceed as follows. We implement a gradient expansion and adopt the following perturbative Ansatz

$$
\begin{aligned}
\varphi_{k}(\tau)= & -i \frac{e^{-i k \tau}}{\sqrt{2 k^{3}}} z(\tau) \frac{H_{0}}{c_{0}} \mathcal{C}_{1}\left[1+i k \tau+\left(i k \tau_{0}\right)^{2} A_{(2)}(\tau)+\left(i k \tau_{0}\right)^{3} A_{(3)}(\tau)+\ldots\right] \\
& -i \frac{e^{i k \tau}}{\sqrt{2 k^{3}}} z(\tau) \frac{H_{0}}{c_{0}} \mathcal{C}_{2}\left[1-i k \tau+\left(-i k \tau_{0}\right)^{2} A_{(2)}(\tau)+\left(-i k \tau_{0}\right)^{3} A_{(3)}(\tau)+\ldots\right],
\end{aligned}
$$

which has the property to match to the standard de Sitter mode function when the functions $A_{(n)}=0$. Recall that the overall factor $H_{0} / c_{0}$ [introduced in Eq. (2.3)] accommodates distinct possibilities for the nature of fluctuation $\psi_{k}$ (scalar fluctuation, Sasaki-Mukhanov variable, tensor modes etc). The previous Ansatz contains a new timescale $\tau_{0}$ that we use for defining a convenient dimensionless parameter $\Delta \tau / \tau_{0}$. The final results are independent from $\tau_{0}$ though.

In what comes next, the dimensionless functions $A_{(n)}$ entering Eq. (2.11) are chosen so to ensure that they vanish for $\tau \leq \tau_{1}$. This implies that we get the general de Sitter solution of Eq. (2.4) at early times: if we wish to match with the Bunch-Davies vacuum, then $\mathcal{C}_{1}=1$ and $\mathcal{C}_{2}=0$; but for our discussion we keep arbitrary values for these constants. The quantity $\tau_{0}$ is the reference scale (2.3). Similar perturbative approaches have been developed in other works, as we briefly review in Sec. II F.

Plugging the Ansatz (2.11) into Eq. (2.10), we analyze the problem in terms of a gradient expansion in powers of $k$. We find the following system of differential equations in the time coordinate, valid for each power of $k$ bigger or equal to 2 (a prime indicates derivative along time):

$$
\begin{gathered}
{\left[\frac{\omega(\tau)}{\tau^{2}} \tau_{0}^{2} A_{(2)}^{\prime}(\tau)\right]^{\prime}=\frac{\omega^{\prime}(\tau)}{\tau},} \\
{\left[\frac{\omega(\tau)}{\tau^{2}}\left(\tau_{0} A_{(n)}^{\prime}(\tau)-A_{(n-1)}(\tau)\right)\right]^{\prime}} \\
=\frac{\omega(\tau) A_{(n-1)}^{\prime}(\tau)}{\tau^{2}}, \text { for } n>2 .
\end{gathered}
$$

When $\omega(\tau)$ is a constant we learn that $A_{(n)}=0$ is a consistent solution of the previous system of equations; on the other hand, any nontrivial profile of $\omega(\tau)$ switches on the $A_{(n)}$-contributions.

In fact, a formal solution for $A_{(2)}$ in Eq. (2.12) is given by the expression

$$
\tau_{0}^{2} A_{(2)}(\tau)=\int_{-\infty}^{\tau} d \tau_{a} \frac{\tau_{a}^{2}}{\omega\left(\tau_{a}\right)}\left(\int_{-\infty}^{\tau_{a}} d \tau_{b} \frac{\omega^{\prime}\left(\tau_{b}\right)}{\tau_{b}}\right)
$$

The extremes of integration are chosen to satisfy the conditions we wish to impose: when $\tau \leq \tau_{1}$, then $A_{(2)}(\tau)=0$. Alternatively, we can set the lower limits of 
the integral to $\tau_{1}$, and $\operatorname{set} A_{(2)}(\tau)=0$ for $\tau \leq \tau_{1}$. This choice of boundaries completely fix the solution for $A_{(2)}(\tau)$.

Passing to higher $n$ 's, a formal solution for $A_{(n)}$ in Eq. (2.13) is given by the expression

$$
\begin{aligned}
\tau_{0} A_{(n)}(\tau)= & \int_{-\infty}^{\tau} d \tau_{a} A_{(n-1)}\left(\tau_{a}\right) \\
& +\int_{-\infty}^{\tau} d \tau_{a} \frac{\tau_{a}^{2}}{\omega\left(\tau_{a}\right)}\left(\int_{-\infty}^{\tau_{a}} d \tau_{b} \frac{\omega\left(\tau_{b}\right) A_{(n-1)}^{\prime}\left(\tau_{b}\right)}{\tau_{b}^{2}}\right) .
\end{aligned}
$$

Again, the extremes of integration are chosen to ensure that $A_{(n)}(\tau)=0$ for $\tau \leq \tau_{1}$.

This is the first new result of our work: we determined the most general formal solutions for the functions $A_{(n)}$. These formal solutions satisfy the correct boundary conditions that completely specify the system. If we were able to systematically perform all the integrals in Eqs. (2.14), (2.15), we would get the full solution for the Eq. (2.10) we wish to solve. We now exploit the fact that the epoch of slow-roll violation lasts a short time interval $\Delta \tau=\tau_{2}-\tau_{1}$ and we compute the exact expressions for the $A_{(n)}(n \geq 2)$ at leading order in $\Delta \tau / \tau_{0}$.

\section{Solving the equations at leading order in $\Delta \tau / \tau_{0}$}

Working at leading order in a Taylor expansion in $\Delta \tau / \tau_{0}$, we can explicitly determine the solutions for the functions $A_{(n)}$ (with $n \geq 2$ ). Since $\Delta \tau$ is small, we can Taylor expand, and keep the first nonvanishing term. Hence, for the function $A_{(2)}$ we find

$A_{(2)}\left(\tau_{2}\right)=A_{(2)}\left(\tau_{1}\right)+A_{(2)}^{1}\left(\tau_{1}\right) \Delta \tau+\frac{1}{2} A_{(2)}^{2}\left(\tau_{1}\right) \Delta \tau^{2}+\ldots$

where the upper index in boldface indicates from now on the order of derivative. It is clear that $A_{(2)}\left(\tau_{1}\right)=0$. Then using $^{3}$ formula (2.14) we get

$\tau_{0}^{2} A_{(2)}^{1}(\tau)=\frac{\tau^{2}}{\omega(\tau)} \int_{-\infty}^{\tau} d \tau_{a} \frac{\omega^{\prime}\left(\tau_{a}\right)}{\tau_{a}} \Rightarrow A_{(2)}^{1}\left(\tau_{1}\right)=0$,

due to the fact that for $\tau<\tau_{1}$ we have $\omega^{\prime}=0$. On the other hand, the second derivative has a nonvanishing contribution, since

\footnotetext{
${ }^{3}$ These arguments are valid in the physically reasonable assumption that the derivatives of $\omega(\tau)$ do not diverge within the small interval $\Delta \tau$, so that $\lim _{\Delta \tau \rightarrow 0} \omega^{\mathbf{n}}\left(\tau_{1}\right) \Delta \tau=0$.
}

$$
\begin{aligned}
\tau_{0}^{2} A_{(2)}^{2}(\tau) & =\left[\partial_{\tau}\left(\frac{\tau^{2}}{\omega(\tau)}\right) \int_{-\infty}^{\tau} d \tau_{a} \frac{\omega^{\prime}\left(\tau_{a}\right)}{\tau_{a}}\right]+\frac{\tau}{\omega(\tau)} \omega^{\prime}(\tau) \\
\Rightarrow \tau_{0}^{2} A_{(2)}^{2}\left(\tau_{1}\right) & =\frac{\tau_{1} \omega^{\prime}\left(\tau_{1}\right)}{\omega\left(\tau_{1}\right)} .
\end{aligned}
$$

Then, we define

$$
\tau_{0}^{2} A_{(2)}^{2}\left(\tau_{1}\right) \equiv \alpha=\left.\frac{d \ln \omega(\tau)}{d \ln \tau}\right|_{\tau=\tau_{1}} .
$$

This implies that the leading contribution to the Taylor series in an expansion in $\frac{\Delta \tau}{\tau_{0}}$ is

$$
A_{(2)}\left(\tau_{2}\right) \simeq \frac{\alpha}{2} \frac{\Delta \tau^{2}}{\tau_{0}^{2}} .
$$

We can proceed in the same spirit with $A_{(3)}$. Choosing $n=3$ in Eq. (2.15) and taking the first derivative along $\tau$, we find

$\tau_{0} A_{(3)}^{\mathbf{1}}(\tau)=A_{(2)}(\tau)+\frac{\tau^{2}}{\omega(\tau)} \int_{-\infty}^{\tau} d \tau_{a} \frac{\omega\left(\tau_{a}\right) A_{(2)}^{\mathbf{1}}\left(\tau_{a}\right)}{\tau_{a}^{2}}$.

Simple manipulations, using also the previous results (2.18)-(2.20) obtained for $A_{(2)}\left(\tau_{1}\right)$ and its derivatives, lead to

$$
\begin{gathered}
A_{(3)}^{\mathbf{0}}\left(\tau_{1}\right)=A_{(3)}^{\mathbf{1}}\left(\tau_{1}\right)=A_{(3)}^{2}\left(\tau_{1}\right)=0, \\
\tau_{0}^{3} A_{(3)}^{\mathbf{3}}\left(\tau_{1}\right)=2 \alpha .
\end{gathered}
$$

Proceeding recursively, one finds that the first nonzero derivative of $A_{(n)}$ evaluated at $\tau=\tau_{1}$ is the $n$ th, with

$$
\tau_{0}^{n} A_{(n)}^{\mathbf{n}}\left(\tau_{1}\right)=2 \tau_{0}^{n-1} A_{(n-1)}^{\mathbf{n}-\mathbf{1}}\left(\tau_{1}\right)=2^{n-2} \alpha .
$$

This implies that for each $n$, the leading order in the corresponding Taylor expansion is

$$
A_{(n)}\left(\tau_{2}\right) \simeq \frac{2^{n-2}}{n !} \alpha \frac{\Delta \tau^{n}}{\tau_{0}^{n}}, \quad \text { for any } n \geq 2 .
$$

Hence, we have been able to solve at each order in $n$ the system of equations (2.12), (2.13), determining for each $n$ the leading order contribution to the functions $A_{(n)}$, in a Taylor expansion in the quantity $\Delta \tau / \tau_{0}$. The solutions depend on the parameter $\alpha$-defined in (2.19) - characterising the effective slope of the pump field $z(\tau)$, as well as on the duration $\Delta \tau$ of the non-slow-roll era.

How much are we losing neglecting the higher order terms in each of the Taylor expansions discussed above? Consider for example $A_{2}(\tau)$. Including the next-to-leading contribution to the Taylor expansion we find a correction to Eq. (2.20) which reads 
$A_{(2)}\left(\tau_{2}\right)=\frac{\alpha}{2} \frac{\Delta \tau^{2}}{\tau_{0}^{2}}\left[1+\frac{\Delta \tau}{3 \tau_{1}}\left(2-\alpha+\frac{d \ln \alpha}{d \ln \tau}\right)\right]+\ldots$

where all quantities in the right-hand-side of the previous expressions are evaluated at $\tau_{1}$. More generally, all the remaining corrections are weighted by powers of $\Delta \tau / \tau_{1}$. We impose the hierarchy $\left|\Delta \tau / \tau_{1}\right| \ll 1$ : this corresponds to take a small parameter $\sigma$ in Eq. (2.9), and this choice is motivated by the short duration of the non-slow-roll epoch with respect with the timescale $\left|\tau_{1}\right|$. (Instead, the timeparameter $\tau_{0}$ can be selected so that the ratio $\Delta \tau / \tau_{0}$ is parametrically larger than $\Delta \tau / \tau_{1}$, so that the leading term in the expansion is non-negligible.) We also assume that the small quantity $\Delta \tau / \tau_{1}$ in Eq. (2.26) is not much amplified by enhancing factors, as the ones contained in the round brackets of Eq. (2.26) in the limit of large parameter $\alpha$. This procedure defines a perturbative scheme that includes only the leading contributions weighted by powers of $\left(\Delta \tau / \tau_{0}\right)^{n}$ in (2.25), while it neglects all the other terms in the Taylor series.

\section{E. Resumming the series, and the leading expression for the mode function}

We can plug these results in the solution for the mode function $\psi_{k}=\varphi_{k} / z(\tau)$, evaluating it at any time $\tau$ within the interval $\tau_{1} \leq \tau \leq \tau_{2}$ when a violation of slow-roll conditions occurs. As an example, we focus on $\tau \rightarrow \tau_{2}$, i.e., toward the end of the non-slow-roll era. Setting $\mathcal{C}_{1}=1$ and $\mathcal{C}_{2}=0$ in Eq. (2.11), so to select a Bunch-Davies vacuum at early times, we get

$$
\begin{aligned}
\psi_{k}\left(\tau_{2}\right) & \sim-i \frac{e^{-i k \tau_{2}}}{\sqrt{2 k^{3}}} \frac{H_{0}}{c_{0}}\left[1+i k \tau_{2}+\left(i k \tau_{0}\right)^{2} A_{(2)}\left(\tau_{2}\right)+\left(i k \tau_{0}\right)^{3} A_{(3)}\left(\tau_{2}\right)+\ldots\right], \\
& =-i \frac{e^{-i k \tau_{2}}}{\sqrt{2 k^{3}}} \frac{H_{0}}{c_{0}}\left[1+i k \tau_{2}-\frac{\alpha}{4}-\frac{1}{2} i k \alpha \Delta \tau+\frac{\alpha}{4} \sum_{n=0}^{\infty} \frac{(2 i k)^{n}}{n !} \Delta \tau^{n}\right] .
\end{aligned}
$$

We can resum exactly the series, and get (recall that $\Delta \tau=\tau_{2}-\tau_{1}$ )

$$
\psi_{k}\left(\tau_{2}\right)=-i \frac{e^{-i k \tau_{2}}}{\sqrt{2 k^{3}}} \frac{H_{0}}{c_{0}}\left[\left(1+i k \tau_{2}\right)-\frac{\alpha}{4}\left(1+2 i k \Delta \tau-e^{2 i k \Delta \tau}\right)\right],
$$

for the mode function at the end of the non-slow-roll epoch.

The previous formula can be easily generalized to any value of $\mathcal{C}_{1,2}$-hence allowing for a richer mode evolution or a more general vacuum preceding the non-slow-roll epoch-leading to

$$
\begin{aligned}
\psi_{k}\left(\tau_{2}\right)= & -i \frac{e^{-i k \tau_{2}}}{\sqrt{2 k^{3}}} \mathcal{C}_{1} \frac{H_{0}}{c_{0}}\left[\left(1+i k \tau_{2}\right)-\frac{\alpha}{4}\left(1+2 i k \Delta \tau-e^{2 i k \Delta \tau}\right)\right] \\
& -i \frac{e^{i k \tau_{2}}}{\sqrt{2 k^{3}}} \mathcal{C}_{2} \frac{H_{0}}{c_{0}}\left[\left(1-i k \tau_{2}\right)-\frac{\alpha}{4}\left(1-2 i k \Delta \tau-e^{-2 i k \Delta \tau}\right)\right] .
\end{aligned}
$$

These relatively simple formulas are at the basis of our analytical considerations. The mode function (2.28) (or (2.29) cannot be recast in terms of the general solution (2.4) for massless modes in de Sitter space with a simple profile (2.3) for the pump function. The correction to the "pure de Sitter" solution of Eq. (2.5) is weighted by the dimensionless parameter $\alpha$ within the square parenthesis. The results so far have their points of interest and their limitations:

(i) The mode function (2.28) modifying the de Sitter solution is extremely simple. It depends on the effective parameter $\alpha$, which condenses in a single quantity any deviation with respect to the de Sitter case, and on the duration $\Delta \tau$ of the non-slow-roll epoch. It can be applied to parametrize different scenarios with brief violations of slow-roll conditions. For the case of ultra-slow-roll expansion, one has $\omega(\tau) \propto \tau^{6}$ and $\alpha=6$, but also other values are allowed during non-slow-roll inflation, depending on the scenarios. ${ }^{4}$ In our study, $\alpha$ is considered a free parameter, that can assume large values. The possibility to study the system in terms of very few effective parameters has its clear advantages. For example, next we show that we can combine our

\footnotetext{
${ }^{4}$ Appendix A discusses a toy model of single-field inflation with an arbitrarily fast decreasing pump field, by suitably tuning the inflationary potential. This shows that, at least in principle, any value of $\alpha$ can be achieved in single-field inflation.
} 
results and get universal formulas connecting properties of the power spectrum as a function of the scale, which are independent from specific models.

(ii) On the other hand, it is important to stress that we are truncating each of the Taylor series at the first nonvanishing contribution, and we are not always ensured that the following terms are smaller than the first in all models under consideration. In the previous Sec. II D we identified a perturbative procedure that ensures that our truncation leads to reliable results, if the non-slow-roll period $\Delta \tau$ is short, and the system satisfies appropriate conditions. [See in particular the discussion after Eq. (2.26).] On the other hand, models can exist where $\Delta \tau$ is not negligibly small: the higher order, next-to-leading Taylor corrections to our results can be relevant, if they are weighted by large coefficients that amplifies them. In our investigation we do not enter into explicit analysis of specific models, and we assume to work within systems where our perturbative approach is valid, and our approximations correct. In general, we expect that our perturbative scheme works best when the growth of the spectrum happens quickly through sudden variations of the slow-roll parameters.

\section{F. Brief comparison with previous analytical analysis of the spectrum}

Before starting to analytically explore the consequences of Eq. (2.28), we conclude this section comparing our procedure with other analytic approaches proposed in the literature to study the dynamics of fluctuations in inflationary scenarios containing phases of non-slow-roll evolution.

The work [10] uses an analytical method based on junction conditions, and requires the knowledge of mode functions during the nonattractor era. [10] models the nonattractor evolution in terms of a combination of phases, each with constant slow-roll parameter $\epsilon_{2}$ (that can possibly be large, up to order $\left.\left|\epsilon_{2}\right| \sim \mathcal{O}(10)\right)$ and small slow-roll parameter $\epsilon_{1}$. For example, choosing $\epsilon_{2}=-6$ corresponds to ultra-slow-roll (USR) inflation. The mode functions computed in USR and de Sitter phases as $\psi_{k}^{\mathrm{USR}}(\tau) \propto a^{3}(\tau) \psi_{k}^{\mathrm{dS}}(\tau)$, hence during USR the amplitude of the spectrum of fluctuations grows with time as the sixth power of the scale factor. [Notice that this is different from our mode function (2.28)]. The total duration of non-slow-roll epochs last few e-folds, in order to obtain the necessary amplification of fluctuations. The paper [11] works in the same context and under the same hypothesis of [10], providing an interpretation for the steepest growth of the spectrum in such scenarios, and additionally showing that a prolonged phase of non-slowroll expansion preceding a phase of ultra-slow-roll inflation can enhance the slope of the spectrum of perturbations at superhorizon scales.

An alternative, systematic approach more similar to ours was proposed in [37], solving the mode functions implementing a gradient expansion in powers of $k$ (although using a slightly different Ansatz with respect to us) and studying the role of the decaying mode after horizon crossing. The motivation of this work is to study setup where more drastic departures from slow-roll inflation occur, with the main purpose to find the conditions for obtaining a growth of the spectrum of perturbations. Their methods was preceded by an early paper [27] that proposed to use a $k$-expansion in solving the mode equations in certain scenarios of interrupted inflation. More recently, the methods of [37] were reconsidered in [12] by including higher powers of $k$ in the gradient expansion, so to better understand different slopes of the spectrum of perturbations in the process of its growth toward its peak. None of these works implemented a truncated Taylor expansion in their analysis, as we do here, with the specific purpose to examine what are general features of systems characterized by a short duration of the non-slow-roll epoch.

\section{THE POWER SPECTRUM AND ITS PROPERTIES}

In this section we consider systems where the power spectrum of fluctuations parametrically grows by a factor $\sim 10^{5}$ from large toward small scales. This is a representative large quantity aimed at demonstrating that our formalism can be in principle applied to a variety of cases where the amplitude of perturbations is enhanced during inflationin the scalar and/or in the tensor sectors. ${ }^{5}$ We then investigate the corresponding properties of the spectrum.

We define the power spectrum associated with the mode function $\psi_{k}(\tau)$ of (2.28) at time $\tau$ as

$$
\mathcal{P}_{k}(\tau)=\frac{k^{3}}{2 \pi^{2}}\left\langle\psi_{k}^{*}(\tau) \psi_{k}(\tau)\right\rangle^{\prime}
$$

where the prime indicates a 2-point function understanding the $\delta$-function associated with momentum conservation. Making use of the formulas discussed in the previous section, we can ensure that all mode functions match a Bunch-Davies vacuum at early times.

It is convenient to work with the quantity

$$
\Pi_{k}(\tau) \equiv \frac{\mathcal{P}_{k}(\tau)}{\mathcal{P}_{k \rightarrow 0}(\tau)}
$$

the ratio between spectra evaluated respectively at scale $k$ and at very large scales $k \rightarrow 0$ : this ratio goes to 1 for $k \rightarrow 0$, and makes more manifest the small-scale growth of the spectrum in scenarios with transient violation of slowroll conditions. The spectral index-which generally depends on the scale-is defined as

\footnotetext{
${ }^{5}$ For the production of primordial black holes, the required enhancement of the curvature perturbation spectrum from large to small scales is larger, typically of order $10^{7}$. Such numbers can be obtained in our approach by tuning the available parameters.
} 


$$
n_{s}-1=\frac{d \ln \Pi_{k}(\tau)}{d \ln k} .
$$

An examination of various examples of power spectra profiles for single field inflation discussed in the literature shows two salient features:

(1) While the profile of $\Pi_{k}(\tau)$ as a function of $k$ initially gently decreases as $k$ increase, it typically features a very pronounced dip at some scale $k_{\text {dip }}$, associated with modes that leave the horizon still during the slow-roll era. This fact has been noticed in several papers that numerically analyse the curvature power spectrum in these scenarios, see, e.g., $[2-5,38,39]{ }^{6}$ It would be interesting to obtain quantitative, modelindependent estimates of the position and depth of the dip, and its relation with other features of the spectra. Information on the dip position would allow one to estimate at what scale the spectrum starts to strongly deviate from the amplitude and scaledependence predicted in single-field slow-roll inflation. This can be important when comparing models with the constraints on the large scale spectra obtained from $\mathrm{CMB}$ temperature fluctuations and spectral distortions (see, e.g., [10,40-44]).

(2) Soon after the dip, the spectrum starts to steadily increase as $k$ increases, with a slope that normally does not overcome the limit $n_{s}-1=4$ in single field inflation [10]. This limit has nevertheless been shown to be slightly violated if a phase of nonattractor is immediately preceded by another phase of slow-roll violation [11] (see also [12]). These facts indicate that the behavior of the spectrum during phases of non-slow-roll evolution might have a "memory" of what occurs prior to these phases, and such memory can be stored in the slope of the spectrum. This information is important for studying possible bounds on the process of in the amplitude of perturbations: for example, a stochastic gravitational background induced at second order by enhanced curvature perturbations [45-48] can be constrained today by pulsar timing array observations [49,50].

In what follows we study the previous two points using our formalism, considering two situations. In the first, we assume there is a unique phase of brief non-slow-roll evolution, following and preceding two epochs of (quasi)de Sitter expansion, and we seek for analytic formulas for the properties of the dip and the slope of the corresponding spectrum of fluctuations. In the second, we study scenarios with multiple transient phases of non-slow-roll evolution, connected by epochs of slow-roll expansion: we show that the slope of the resulting spectrum can in principle increase

\footnotetext{
${ }^{6}$ More than one dip can be present at relatively large scales (see, e.g., [39]) but in the examples we know there is always one dip much more pronounced than the others.
}

with respect to scenarios with a single short phase of nonslow-roll evolution.

\section{A. A single phase of slow-roll violation}

\section{The power spectrum during the epoch of slow-roll violation}

We start studying the case of a single phase of non-slowroll inflationary evolution, focusing on the spectrum evaluated at a time $\tau$ between times $\tau_{1}$ and $\tau_{2}$ (for $\tau \leq$ $\tau_{1}$ and $\tau_{2} \leq \tau \leq 0$ we assume standard de Sitter expansion). Evaluating the function $\Pi_{k}(\tau)$ of Eq. (3.4) during such interval and denoting

$$
\Delta \tau_{A}=\tau_{2}-\tau_{1}
$$

we get

$$
\begin{aligned}
\Pi_{k}(\tau)= & 1+\tau^{2} k^{2}+\frac{\alpha}{4}\left[\left(k \Delta \tau_{A}\right)^{2}\left(\alpha-\frac{4 \tau}{\Delta \tau_{A}}\right)\right. \\
& -k \Delta \tau_{A}\left(\alpha-\frac{2 \tau}{\Delta \tau_{A}}\right) \sin \left(2 k \Delta \tau_{A}\right) \\
& \left.+(\alpha-4) \sin ^{2}\left(k \Delta \tau_{A}\right)\right] .
\end{aligned}
$$

The contributions due to non-slow-roll epochs are contained in the second line: they vanish at large scales $k \rightarrow 0$, and become important at small scales. The function (3.4) is an oscillating function with scale-dependent coefficients, which grows unbounded with momentum $k$. This is not surprising since the function is evaluated at time $\tau$ during the non-slow-roll epoch, and we do not consider yet a final phase of de Sitter expansion for $\tau \geq \tau_{2}$ (we will do it in Sec. III A 2).

On the other hand, it is instructive to start briefly studying some features of the spectrum (3.4) evaluated at a time $\tau$ during slow-roll violation, to point out general properties that will be in common with all the more complex cases we will study.

The growth of the power spectrum.-Since there is only a single parameter $\alpha$ which controls any modifications of standard slow-roll formulas, we expect that this parameter controls the growth of the spectrum: the larger $\alpha$ is, the larger the growth. In fact, we find in the limit of large $\alpha$ and small period $\Delta \tau_{A}$ the following expression for the spectrum $\Pi(k)$, evaluated at a scale $k \tau \sim 1$ :

$$
\Pi_{k=1 / \tau}(\tau) \equiv \Pi_{\max } \simeq \frac{\alpha^{2}}{4}\left(\frac{\Delta \tau_{A}}{\tau}\right)^{4}+\ldots
$$

If we wish to gain several orders of magnitude in the amplitude of the spectrum with respect to its large-scale value, we need to choose a large parameter $\alpha$ : for us, as 


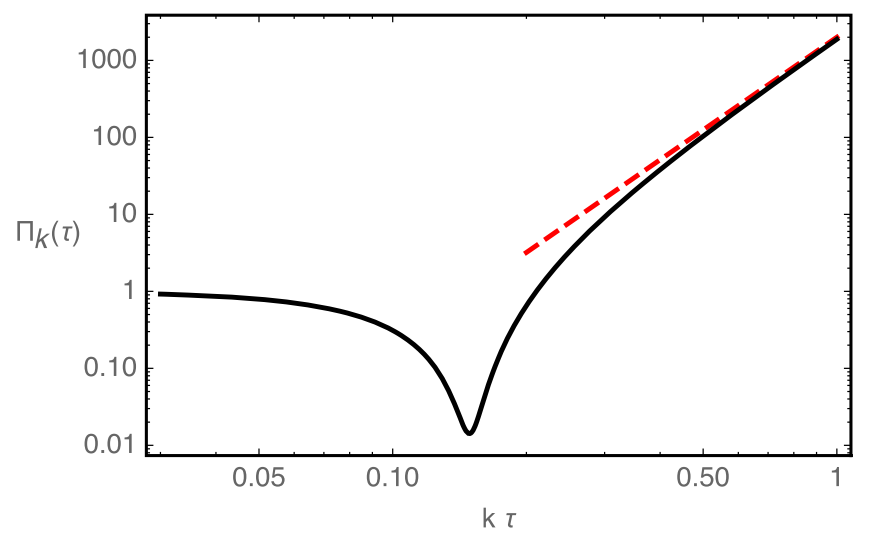

FIG. 2. Plot of the analytic function Eq. (3.4) giving the spectrum $\Pi(k)$ evaluated at time $\tau$ during non-slow-roll violation, versus the dimensionless combination $k \tau$. We choose the representative values $\alpha=1000, \Delta \tau_{A} / \tau=0.3$ for the parameters that enter in Eq. (3.4). The red dashed line has a profile proportional to $(k \tau)^{4}$ and indicates the maximal slope the spectrum can acquire after the dip.

discussed above this parameter is then used as "phenomenological" quantity to control features of the spectrum (in this case its maximal growth), without worrying about predictions of specific models with respect to $\alpha$ (see also the next sections III A 2 and III B for further discussions on this point).

The position and depth of the dip.-Figure 2 shows a dip in the spectrum before a rapid growth: this feature is in common with all the scenarios we study and is known in numerical $[2-5,38,39]$ as well as analytical studies [10,37]. Working in the limit of large parameter $\alpha$, we will analytically understand and analyze the dip position and its properties. We expand function (3.4) at small scales:

$$
\Pi_{k}(\tau)=1-\tau^{2} k^{2}\left(\alpha \frac{\Delta \tau_{A}^{2}}{\tau^{2}}-1\right)+\mathcal{O}\left(\tau^{4} k^{4}\right) .
$$

While for $k \rightarrow 0$ this expression goes to one, for slightly larger values of $k$ we expect the second term to become important, and (if $\alpha$ is positive) to reduce the amplitude of the spectrum. At larger values of $k$ the additional powers $(k \tau)^{4}$ take over, leading to a growth in $k$, and causing the dip we notice in Fig. 2. In fact, we can do better and analytically understand the dip position, since the dip is expected to be located at relatively large scales. We rescale the momentum $k$ by an inverse power of $\alpha$ introducing the variable $x$ as

$$
\Delta \tau_{A} k \equiv \frac{\sqrt{2} x}{\sqrt{\alpha}} .
$$

This Ansatz is motivated by the fact that the dip position occurs at relatively large scales, and it is expected to be controlled by some inverse powers of $\alpha$-being this the only potentially large parameter available. In fact, we can express the spectrum (3.4) as a function of the quantity $x$ defined in Eq. (3.7). By making an expansion for large $\alpha$ we notice that the resulting formula has a leading term, followed by subleading terms that are weighted by inverse powers of the large parameter $\alpha$ :

$$
\begin{aligned}
\Pi_{x}(\tau)= & \left(x^{2}-1\right)^{2}+\frac{x^{2}}{9 \alpha} \frac{18 \tau^{2}+12 x^{2} \Delta \tau_{A}\left(\Delta \tau_{A}-\tau\right)-4 x^{4} \Delta \tau_{A}^{2}}{\Delta \tau_{A}} \\
& +\mathcal{O}\left(\frac{1}{\alpha^{2}}\right) .
\end{aligned}
$$

Hence the value $x=1$ corresponds to the position where spectrum exhibits a dip, being suppressed by the large value of $\alpha$. Since $\alpha$ also controls the total growth of the spectrum, we expect that position and amplitude of the dip can be analytically related with some powers of this quantity. In fact, formulas (3.8) and (3.5) combined together suggest the relations

$\Delta \tau_{A} k_{\mathrm{dip}} \propto\left(\frac{1}{\Pi_{\max }}\right)^{1 / 4}, \quad \Pi_{k=k_{\mathrm{dip}}}(\tau) \propto\left(\frac{1}{\Pi_{\max }}\right)^{1 / 2}$,

that will be made more quantitative in the next sections, once we include the last phase of de Sitter expansion and evaluate all quantities at the end of inflation. We find these formulas potentially interesting since they are independent from $\alpha$, hence they can provide universal, model independent relations between the property of the dip and the maximal growth of the spectrum.

The maximal slope of the spectrum in the final phase of growth.-After the dip, the small-scale spectrum of Fig. 2 starts to steadily grow, with a spectral index whose maximal value is $n_{s}-1=4$, in agreement with the analysis of [10].

Notice that the maximal power of $k$ in the coefficients of the oscillating functions of (3.4) is $k^{2}$ : on the other hand, the oscillating functions have their own scale-dependence that can "reinforce" the slope of the spectrum, allowing for higher powers of $k$. We can understand analytically these facts from our analytic formula (3.4). We focus on small scales far away from the dip, and far from the large scale regime where a large $\alpha$ expansion can be compensated by very small values of $k \tau$. Working at such small scales, we concentrate on the leading order contributions to the spectral index in an expansion in inverse powers of $1 / \alpha$. We find, expanding for large $\alpha$,

$$
\begin{aligned}
n_{s}-1= & \frac{8\left(k \Delta \tau_{A}\right)^{2} \sin ^{2}\left(k \Delta \tau_{A}\right)}{1+2\left(k \Delta \tau_{A}\right)^{2}-\cos \left(2 k \Delta \tau_{A}\right)-2 k \Delta \tau_{A} \sin \left(2 k \Delta \tau_{A}\right)} \\
& +\mathcal{O}\left(\frac{1}{\alpha}\right), \\
= & 4-\frac{4}{9}\left(k \Delta \tau_{A}\right)^{2}+\mathcal{O}\left(\frac{1}{\alpha},\left(k \Delta \tau_{A}\right)^{4}\right) .
\end{aligned}
$$




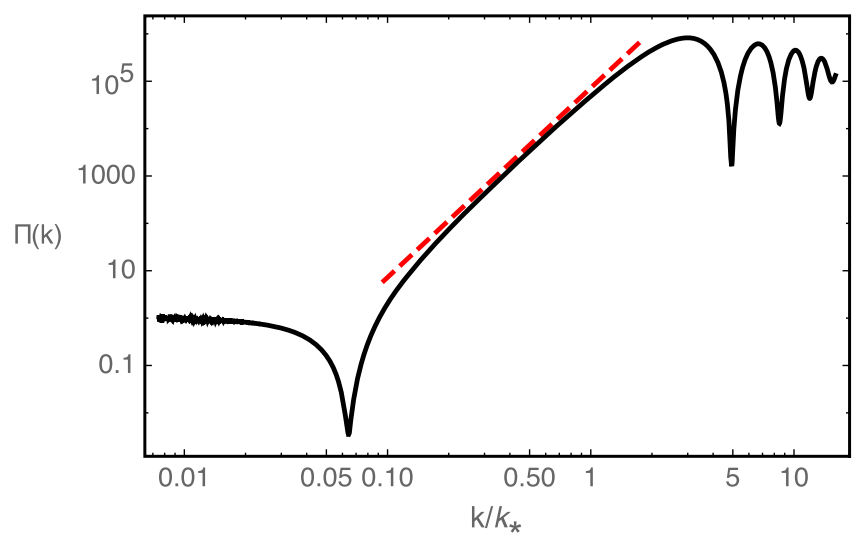

FIG. 3. Plot of the analytic function in Eq. (3.13) giving the spectrum $\Pi(k)$ at the end of inflation versus the dimensionless combination $k / k_{\star}$, with $k_{\star}=\left(\Delta \tau_{A}+\Delta \tau_{B}\right)^{-1}$. We choose the representative values $\alpha=4000, \Delta \tau_{A} / \Delta \tau_{B}=0.2$. The pivot scale $k_{\star}$ is chosen as the scale at which the modes start leaving the horizon during the non-slow-roll era; notice that the dip occurs at smaller values of $k$. The red dashed line has scale-dependent profile proportional to $\left(k / k_{\star}\right)^{4}$.

The oscillating function in the first line of Eq. (3.10) is positive and has a maximal value of 4 , confirming the results of [10-12]. The second line of Eq. (3.10) exhibits this fact more transparently. In fact, although we work at small scales with sizeable $k$, they are not really that small: indeed we expect $k \tau$ to be of order 1 , and $\Delta \tau_{A} / \tau \ll 1$ for a short duration of the non-slow-roll phase. Then, we expand the first line of Eq. (3.10) for small value of $k \tau \Delta \tau_{A} / \tau=k \Delta \tau_{A}$. In the second line of the formula we learn that the maximal spectral index is $n-1 \simeq 4$ in agreement with Fig. 2, and with the works [10-12].

\section{Matching with a final phase of de Sitter expansion}

After pointing out some universal features of the power spectrum indicated by our analytical approach, we make them more quantitative by matching the system studied so far with a final phase of de Sitter expansion from $\tau=\tau_{2}$ to $\tau=0$. For $\tau \leq \tau_{2}$ we have the solution (2.28) for the mode function $\psi_{k}(\tau)$; for $\tau \geq \tau_{2}$ the pump field $z(\tau)$ acquires its "pure-de Sitter" profile $z \propto 1 / \tau$ [see Eq. (2.1)] and the corresponding mode function is given by Eq. (2.4) (depending on the time-independent constants $\mathcal{C}_{1,2}$ ). We can then match solutions (2.28) and (2.4) and their derivatives through Israel matching conditions at the transition time $\tau_{2}$. Assuming that for $\tau \geq \tau_{2}$ we have pure de Sitter evolution up to the end of inflation at $\tau=0$ we can identify

$$
\Delta \tau_{B}=0-\tau_{2}
$$

as the duration of the final de Sitter phase. A straightforward computation gives the following values for the constant (but scale-dependent) parameters $\mathcal{C}_{1,2}$ :

$$
\begin{aligned}
\mathcal{C}_{1}(k)= & 1+\alpha \frac{1-e^{2 i k \Delta \tau_{A}}-2 i k \Delta \tau_{A}\left(1+2 i k \Delta \tau_{B}\right)}{8 k^{2} \Delta \tau_{B}^{2}}, \\
\mathcal{C}_{2}(k)= & -\alpha \frac{e^{2 i k \Delta \tau_{B}}}{8 k^{2} \Delta \tau_{B}^{2}} \\
& \times\left(1-2 i k\left(\Delta \tau_{A}+\Delta \tau_{B}\right)-e^{2 i k \Delta \tau_{A}}\left(1-2 i k \Delta \tau_{B}\right)\right),
\end{aligned}
$$

and the corresponding spectrum $\Pi_{k}(\tau)$ evaluated at the end of inflation, $\tau=0$ results simply

$$
\Pi_{k}(\tau=0)=\left|\mathcal{C}_{1}(k)+\mathcal{C}_{2}(k)\right|^{2} .
$$

Substituting the values of $\mathcal{C}_{1,2}$ the complete expression for $\Pi_{k}(\tau=0)$ is rather cumbersome, but it is straightforward to obtain it combining formulas (3.11), (3.12), and (3.13). We avoid putting such long equations in the text, and represent the result in Fig. 3 for a representative choice of the parameters.

The maximal growth of the spectrum.-The plot 3 represents $\Pi_{k}$ at the end of inflation as function of the ratio $k / k_{\star}$, where we introduce the pivot scale

$$
k_{\star}=\frac{1}{\left(\Delta \tau_{A}+\Delta \tau_{B}\right)},
$$

corresponding to a scale at which a mode starts to leave the horizon during the non-slow-roll epoch. For scales larger than $k_{\star}$ (i.e., $k \leq k_{\star}$ ) the modes leave the horizon during the initial phase of pure de Sitter expansion. The content of Fig. 3 is qualitatively similar to what discussed in Sec. III A 1: it presents a dip in the spectrum at relatively large scales (that left the horizon well earlier than the onset of the non-slow-roll epoch) as well as a maximal slope $n_{s}-1=4$ for the growth of the spectrum after the dip occurs. At very small scales (large $k / k_{\star}$ ) the spectrum asymptotes on average to a constant, and the "oscillatory" behavior is due to our instantaneous matching of the mode function to the pure de Sitter mode solution. It is easy to take the asymptotic limit of the function (3.13) at very small scales. Introducing the dimensionless quantity

$$
\epsilon_{A}=\frac{\Delta \tau_{A}}{\Delta \tau_{A}+\Delta \tau_{B}},
$$

we find the expression

$$
\Pi_{\max }=\left(1+\frac{\alpha}{2} \frac{\Delta \tau_{A}}{\Delta \tau_{B}}\right)^{2}=\left(1+\frac{\alpha}{2} \frac{\epsilon_{A}}{1-\epsilon_{A}}\right)^{2},
$$

for the value of the spectrum at very small scales. This expression has the expected properties for such asymptotic limit: it diverges when $\Delta \tau_{B} \rightarrow 0$ (equivalently $\epsilon_{A} \rightarrow 1$ ), 
reflecting the fact that in absence of the last phase of de Sitter expansion the spectrum grows unbounded. In the limit of large $\alpha$, it scales as $\alpha^{2}$, showing that the parameter $\alpha$ also in this case controls the amplitude of the spectrum.

Two important points are worth emphasizing:

(i) In plotting Fig. 3 we have chosen a very large value of $\alpha$, in order to obtain a spectrum growth of order $\sim 10^{5}$ from large to small scales: in our discussion here $\alpha$ is a tunable parameter that we treat phenomenologically, and we do not discuss possible scenarios giving so large values. On the other hand, if too large values of $\alpha$ turn out to be unpractical, we might think to further break down the time-interval $\Delta \tau_{A}$ of non-slow-roll evolution in smaller "time-bins"each characterized by a smaller $\alpha$-that combined together accumulate the necessary large growth of the spectrum. We briefly explore this possibility in Sec. III B, showing that indeed the resulting profile of the spectrum is similar to Fig. 3 .

(ii) In the example we study the amplitude of the spectrum tends to a constant at small scales after reaching the peak, which is of the same order of the size of the peak [this is why we call it $\Pi_{\max }$ in Eq. (3.16)]. In other studies, the spectrum tends to decrease with $k / k_{\star}$ after the peak have been reached. This depends on the (model-dependent) dynamics and properties of the last stages of the inflationary expansion after the end of the non-slow-roll phase. In what follows, we call $\Pi_{\max }$ the size of the enhancement in the amplitude of the spectrum at around the peak position.

General properties of the dip.-Working on a large $\alpha$ limit, we can study the position and amplitude of the dip making a large- $\alpha$ expansion of the spectrum expressed in terms of the variable $x$ as

$$
\frac{k}{k_{\star}} \equiv \frac{x}{\sqrt{\alpha}},
$$

analogously to the arguments explained around Eq. (3.7). This Ansatz is motivated by the fact that the dip position occurs at relatively large scales, and it is then expected to be controlled by some inverse powers of $\alpha$, the only large parameter available. This Ansatz turns to be correct: in the large $\alpha$ limit, the resulting spectrum is expressed as a part that does not depend on $\alpha$, plus subleading contributions suppressed by powers of $\alpha$-exactly analogously to what found in Eq. (3.8). The part that does not depend on $\alpha$ vanishes at a scale

$$
\frac{k_{\mathrm{dip}}}{k_{\star}}=\frac{\sqrt{3}}{\sqrt{1-\epsilon_{A}} \sqrt{2 \epsilon_{A}+1}} \frac{1}{\left(\Pi_{\max }^{1 / 2}-1\right)^{1 / 2}},
$$

indicating the position of the dip, with $\Pi_{\max }$ given in Eq. (3.16) and $\epsilon_{A}$ in Eq. (3.15). If $\Pi_{\max }$ is large, and we take
$\epsilon_{A} \ll 1-$ consistently with the fact that we take a short duration for the non-slow-roll epoch-the previous relation simplifies giving

$$
\frac{k_{\mathrm{dip}}}{k_{\star}} \simeq \sqrt{\frac{3}{2}} \frac{1}{\prod_{\max }^{1 / 4}},
$$

providing us with a simple, universal relation for the position of the dip as a function of $\Pi_{\max }$, the maximal growth of the spectrum. Notice that $k_{\text {dip }}$ is generally well smaller than $k_{\star}$, the scale when non-slow-roll starts, and at leading order is independent on the actual duration of the short non-slow-roll phase.

Importantly, this relation connects distinct quantities in a way that is independent on the values of the model parameters (as $\alpha$ ) and only depends on physical properties of the spectrum. The depth of the dip is also straightforward to determine: calling $\Pi_{\min }=\Pi_{k=k_{\text {dip }}}(0)$, we find

$$
\begin{gathered}
\Pi_{\min }=\frac{12}{\left(1-\epsilon_{A}\right)\left(\epsilon_{A}+2\right)^{3}} \frac{1}{\left(\Pi_{\max }^{1 / 2}-1\right)}, \\
\simeq \frac{3}{2} \frac{1}{\Pi_{\max }^{1 / 2}}=\frac{k_{\mathrm{dip}}^{2}}{k_{\star}^{2}} .
\end{gathered}
$$

Then also the depth of the dip is related with the value of the maximal growth of the spectrum through the previous universal relation. Figure 4 shows that our analytic formulas (3.19), (3.20) work very well in determining the properties of the dip for the example represented in Fig. 3.

The information we derived, besides being theoretically interesting, is also relevant for phenomenology. Starting from $k_{\text {dip }}$ the spectrum then starts a steady growth toward its maximum. The position of the dip is important being the characteristic scale at which the spectrum profile starts to considerably deviate from slow-roll predictions, and we need

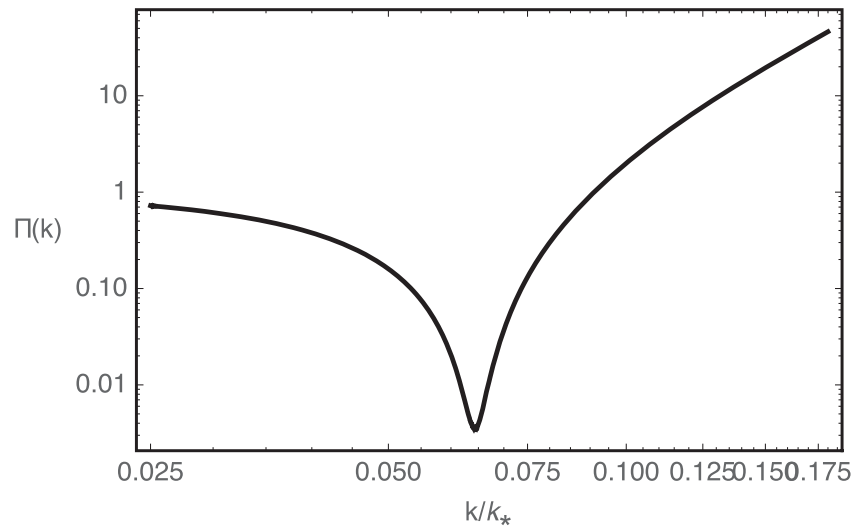

FIG. 4. Zoom on the dip of Fig. 3. We choose the representative values $\alpha=4000, \Delta \tau_{A} / \Delta \tau_{B}=0.2$ for the parameters that enter in Eq. (3.4). Our formulas (3.19), (3.20) give $k / k_{*}=0.064$ while $\Pi\left(k_{\text {dip }}\right)=0.0035$, in agreement with the plot. 
to ensure its profile is consistent with large-scale constraints from CMB and spectral distortions [10,40,42-44].

Our relations (3.19), (3.20) are obtained under the hypothesis that before the non-slow-roll epoch the universe is in a de Sitter phase, with a mode function for perturbations matching a Bunch-Davies vacuum at early times. We derived this formula in a limit of short non-slow-roll phase, truncating the Taylor series as explained in Sec. II. We connect our non-slow-roll phase abruptly with a phase of de Sitter expansion toward the end of inflation. Despite these many hypothesis, these formulas match well analogous formulas determined in the literature using other analytic methods. For example, the authors of [10] study the growth of the spectrum in a setup including a phase of ultra-slowroll inflation, making the hypothesis that in the epoch of slow-roll violation the slow-roll parameter $\epsilon_{2}$ can be large but constant, and analyzing the system using Israel matching conditions. They find for the position of the dip an expression ( $N_{\text {USR }}$ being the number of e-folds of ultraslow-roll expansion)

$$
\frac{k}{k_{\star}} \simeq \sqrt{\frac{5}{4}}\left(\exp \left[6 N_{\mathrm{USR}}\right]\right)^{-1 / 4} \propto \sqrt{\frac{5}{4}} \Pi_{\max }^{-1 / 4},
$$

in good overall agreement with our formula (3.19).

Given these preliminary results, it would be interesting to study in more general terms how precise these formulas can be in fully realistic models with non-slow-roll behavior, possibly including corrections associated with a longer duration of the non-slow-roll phase, and a more realistic matching to the final phase of slow-roll expansion. We leave these investigations to future work. In Sec. III B we instead study how these results are modified in the presence of multiple short phases of non-slow-roll evolution.

The slope of the spectrum.-Figure 2 shows that the maximal slope the spectrum is $n_{s}-1=4$, as found in [10]. The work [11] found an interesting physical explanation of this fact in terms of the behavior of the mode functions during epochs of non-slow-roll, under the hypothesis that the (possibly large) slow-roll parameters are constant. Our approach provides an alternative analytic understanding of this behavior without any assumption on the slow-roll parameter behavior, using the same arguments we developed in Sec. III A 1. We focus in a region of scales $k$ well after the dip, when the large- $\alpha$ contributions to the spectral index do not risk to be compensated by extremely small coefficients of $k / k_{\star}$. In a region of $0.1<k / k_{\star}<1$ we can expand the spectral index for large $\alpha$, small $k / k_{\star}$, and small $\epsilon_{A}$, finding

$$
n_{s}-1=4-\frac{k^{2}}{30 k_{\star}^{2}}\left(12-12 \epsilon_{A}+7 \epsilon_{A}^{2}\right)+\ldots
$$

confirming the maximal slope $n_{s}-1=4$.
It is interesting to ask whether we can overcome this threshold within single-field inflation. The work [11] (see also [12]) shown that if a phase of nonattractor inflation is preceded by a prolonged epoch of non-slow-roll evolution, then the growth of the spectrum can increase up a power $k^{5} \ln k$, indicating that the slope of the spectrum during nonslow-roll epoch retains some sort of "memory" of previous phases of evolution of the pump field $z(\tau)$. The finding of [11] was obtained by making a perturbative expansion of solutions of mode functions in regime of constant (possibly large) slow-roll parameters.

We wish to elaborate more on this topic in the next section using our approach. We shall learn that indeed the spectrum can grow steeper, possibly even with larger powers than what has been found so far.

\section{B. Multiple non-slow-roll epochs}

In this section, we investigate whether multiple short phases of non-slow-roll evolution, nested with epochs of de Sitter expansion, can change the results of the previous section. The possibility of many phases of evolution that deviate from slow-roll during the inflationary period might be realized in scenarios with several inflection points in the inflationary potential, as in axion inflation models which include subleading nonperturbative corrections (see, e.g., [39,51-54]), or models based on axion monodromy $[55,56]$, or scenarios where the inflationary potential has several jumps and discontinuity in its first derivatives $[31,32]$. In any case, regardless of possible concrete constructions and models, we aim to study here some phenomenological consequences of this possibility. In Appendix B we support the results of this section with other methods.

As mentioned above, one of our motivations is the work [11] (see also [12]) that suggests that phases of non-slowroll preceding an epoch of nonattractor can change the slope of the spectrum. Intuitively, a more pronounced slope of the spectrum can be heuristically understood from our formulas. The results of section III A 2 indicate that the constant quantities $\mathcal{C}_{1,2}$ characterizing the de Sitter mode function (2.4) in the de Sitter phase following the non-slowroll epoch store a rich $k$-dependence, that leads to the $k$-dependent profile of the complete spectrum of fluctuations when evaluated toward the end of inflation-see indeed Eqs. (3.11), (3.12). If - before ending inflation-we enter in a new, second phase of non-slow-roll evolution, we can use the de Sitter mode function with coefficients $\mathcal{C}_{1,2}$ of Eqs. (3.11), (3.12) as a basis for determining the mode function during the non-slow-roll epoch. This fact can drastically change the resulting profile of the final spectrum, which accumulates both the $k$-dependence associated with the second non-slow-roll phase, as well as the $k$-dependence already stored in the "initial conditions" governing $\mathcal{C}_{1,2}$. The final slope of the spectrum can then retain memory of all phases of non-slow-roll expansion. 
As a concrete realization, we consider a multiple-phase system consisting on the following epochs (recall that we work with negative values of $\tau$ ):

(0) $-\infty \leq \tau \leq \tau_{1}$ : standard de Sitter expansion, with de Sitter mode function (2.4) and Bunch-Davies vacuum $\left(\mathcal{C}_{1}=1, \mathcal{C}_{2}=0\right)$.

(1) $\tau_{1} \leq \tau \leq \tau_{2}$ : first phase of non-slow-roll, described by a mode function (2.28) with characteristic parameter $\alpha$. The duration of this short phase is $\Delta \tau_{A}=\tau_{2}-\tau_{1}$.

(2) $\tau_{2} \leq \tau \leq \tau_{3}$ : an intermediate phase of de Sitter expansion, with de Sitter mode function (2.4), and parameters $\mathcal{C}_{1,2}$ determined by matching conditions as in eqs (3.11), (3.12). The duration of this phase is $\Delta \tau_{B}=\tau_{3}-\tau_{2}$.

(3) $\tau_{3} \leq \tau \leq \tau_{4}$ : second phase of non-slow-roll, described by a mode function (2.29) with characteristic parameter $\beta$. The duration of this short phase is $\Delta \tau_{C}=\tau_{4}-\tau_{3}$.

(4) $\tau_{4} \leq \tau \leq 0$ : last phase of de Sitter expansion, with de Sitter mode function (2.4), and parameters $\mathcal{C}_{1,2}$ determined by matching conditions. This phase concludes inflation. The duration of this epoch is $\Delta \tau_{D}=-\tau_{4}$.

Our formalism allows us to analytically obtain the mode functions and the power spectrum at the end of inflation, at least with the help of some computer program for manipulating algebraic expressions. We do not write the cumbersome final expression for the spectrum at the end of inflation; instead we represent it in Fig. 5 for distinct durations of the intermediate de Sitter phase $\Delta \tau_{B}$. In representing the plots, we choose as pivot scale

$$
k_{\star}=\left(\Delta \tau_{A}+\Delta \tau_{B}+\Delta \tau_{C}+\Delta \tau_{D}\right)^{-1}
$$

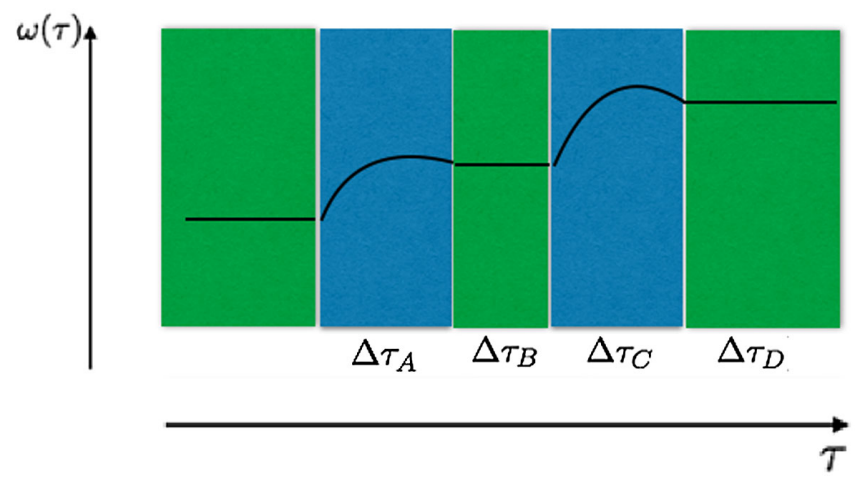

FIG. 5. Pictorial representation of the distinct phases of evolution in the example we consider in this section, extending the examples of Fig. 1 to the case of multiple non-slow-roll epochs. Recall that the function $\omega(\tau)$ controls departures of the pump field $z(\tau)$ from its de Sitter profile [see Eq. (2.7)]. Two phases of non-slow-roll evolution (in blue) are interrupted by a de Sitter epoch (in green). De Sitter expansion also characterizes the epochs preceding and following the non-slow-roll periods. corresponding to the scale where modes start leaving the horizon when the first non-slow-roll phase begins.

Before starting any quantitative analysis, we can point out interesting features of these plots:

(i) First, the upper left plot of Fig. 6 shows a spectrum profile that is qualitatively similar to the plots we found in the previous section, see, e.g., Fig. 3. This plot corresponds to a short duration of the intermediate de Sitter phase between the two non-slow epochs. The spectrum is enhanced by a factor of order $\sim 10^{5}$ from large to small scales, with values of the parameters $\alpha, \beta$ that are one order of magnitude smaller than the ones we considered in Sec. III A 1. This suggests that we might study inflationary systems containing longer phases of non-slow-roll evolution by "breaking down" such non-slow-roll phases in small time bins, that can be then joined together to get the final result. In each bin the pump field profile can be faithfully characterized with few parameters, and the perturbative expansion developed in Sec. II could be applied more reliably. We leave an analysis of this possibility to future work.

(ii) The behavior of the spectrum profile in the plot at the bottom of Fig. 6 is instead different with respect to what we have found so far. It corresponds to a system with a prolonged phase of de Sitter evolution, nested between the two epochs of non-slow-roll expansion. After the initial dip, the spectrum of Fig. 6 grows few orders of magnitude, before meeting another intermediate dip. After the second dip, the spectrum encounters a more enhanced phase of growth, with an amplification by many orders of magnitude, which is characterized by a more pronounced slope. In what follows we will analyze this case, since it can offer opportunities to increase the slope of the perturbation spectrum in single-field inflation.

\section{The maximal growth of the power spectrum}

A straightforward computation analog to the ones we pursued in the previous section leads to the following small-scale (large $k / k_{\star}$ ) limit for the spectrum evaluated at the end of inflation

$\Pi_{\max }=\left(\frac{\left(\beta \Delta \tau_{C}+2 \Delta \tau_{D}\right)\left(\alpha \Delta \tau_{A}+2\left(\Delta \tau_{B}+\Delta \tau_{C}+\Delta \tau_{D}\right)\right)}{4 \Delta \tau_{D}\left(\Delta \tau_{B}+\Delta \tau_{C}+\Delta \tau_{D}\right)}\right)^{2}$.

This quantity represents the maximal growth of the spectrum: setting $\Delta \tau_{B}=\Delta \tau_{C}=0$ one finds the same formula of Eq. (3.16) since we reduce to the case of Sec. III A 1 . In the limit of large $\alpha, \beta$ it simplifies to the expression 

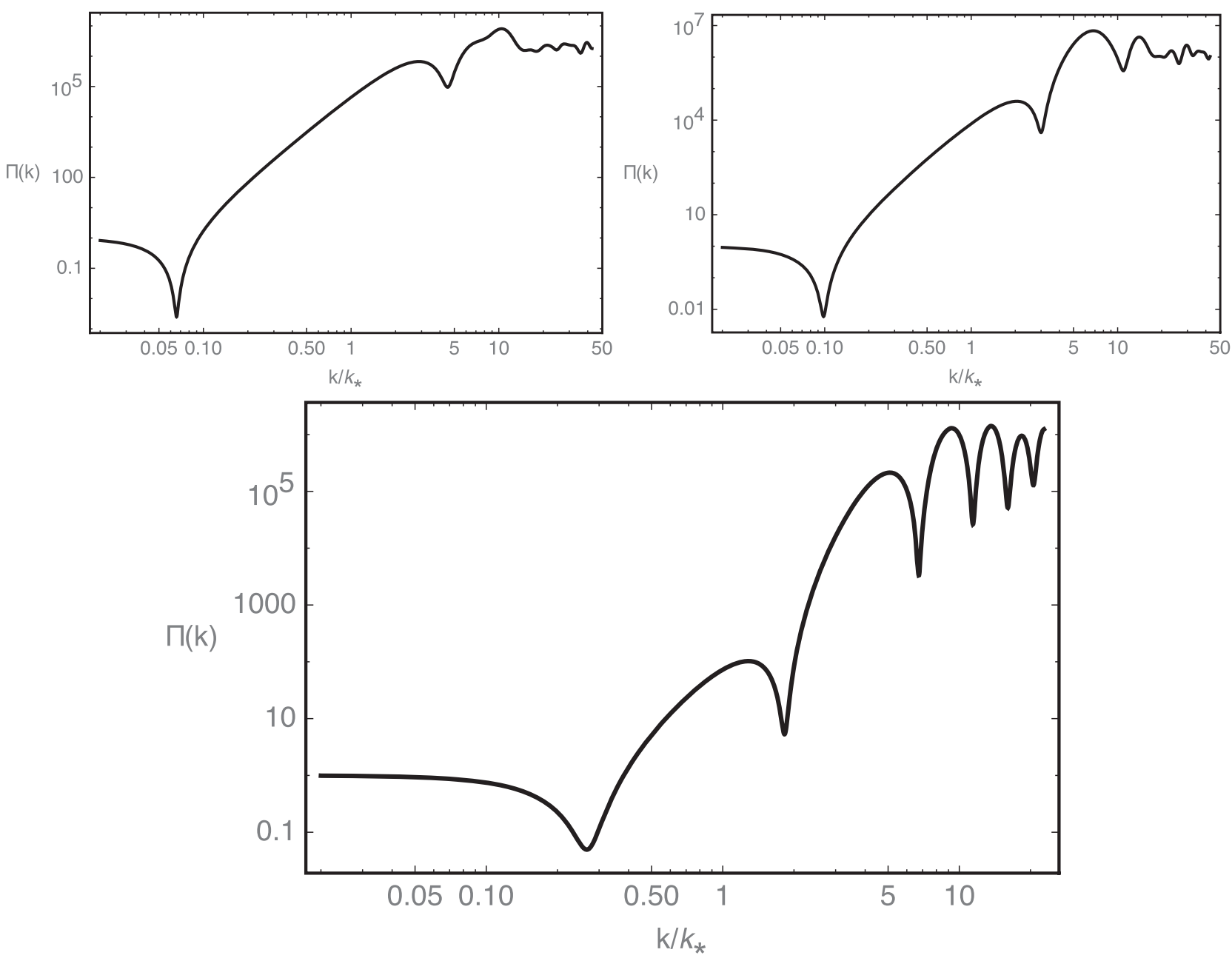

FIG. 6. Three examples of profile of the fluctuation spectrum for the system discussed in this section. The three plots differ for the duration of the intermediate de Sitter phase between the two phases of non-slow-roll evolution. We choose the parameters $\alpha=250$, and $\beta=200$. The duration of the non-slow-roll phases is $\Delta \tau_{A}=\Delta \tau_{C}=0.2$, and the final de Sitter phase lasts $\Delta \tau_{D}=0.7$. For what respect the intermediate de Sitter phase we choose respectively: Top left $\Delta \tau_{B}=0.2$; Top right $\Delta \tau_{B}=0.5$; Bottom $\Delta \tau_{B}=2.0$.

$$
\Pi_{\max }=\frac{\alpha^{2} \beta^{2}}{16}\left(\frac{\Delta \tau_{A} \Delta \tau_{B}}{\Delta \tau_{D}\left(\Delta \tau_{B}+\Delta \tau_{C}+\Delta \tau_{D}\right)}\right)^{2} .
$$

The amplitude of the spectrum is now given by the product of the squares of both parameters $\alpha$ and $\beta$. This is why we can amplify the amplitude of the spectrum by a factor $\sim 10^{5}$ with smaller values of the phenomenological parameters $\alpha, \beta$ with respect to section III A 1 .

\section{Properties of the first dip}

We can determine the position of the first, pronounced dip in plots 6 by rescaling variables as done in the previous section around Eq. (3.17):

$$
\frac{k}{k_{\star}} \equiv \frac{x}{\sqrt{\alpha} \sqrt{\beta}},
$$

and take the large $\alpha, \beta$ limit of the spectrum. Defining

$$
\epsilon_{A}=k_{\star} \Delta \tau_{A}
$$

and analogously for the other time intervals, we find the following expression for the position of the dip:

$$
\frac{k_{\mathrm{dip}}}{k_{\star}} \simeq\left[\frac{3\left(\epsilon_{B}+\epsilon_{D}\right)^{2}}{4 \epsilon_{C} \epsilon_{D}^{2}\left(\epsilon_{B}+2 \epsilon_{D}\right)}\right]^{1 / 2} \frac{1}{\Pi_{\max }^{1 / 4}} .
$$

We derived this expression in the limit of large $\Pi_{\max }$, and of short duration of the non-slow-roll phases. This formula is slightly less accurate in determining the position of the dip with respect to the formulas we found in the previous section, due to the fact that in this more complex system the dip width is larger than in the single non-slow-roll phase. But in studying various examples we find that it is accurate up to a factor of order one in determining $k_{\text {dip }}$. What is interesting is that the dip position in this case is tunable: the position again scales as the inverse of the fourth root of 

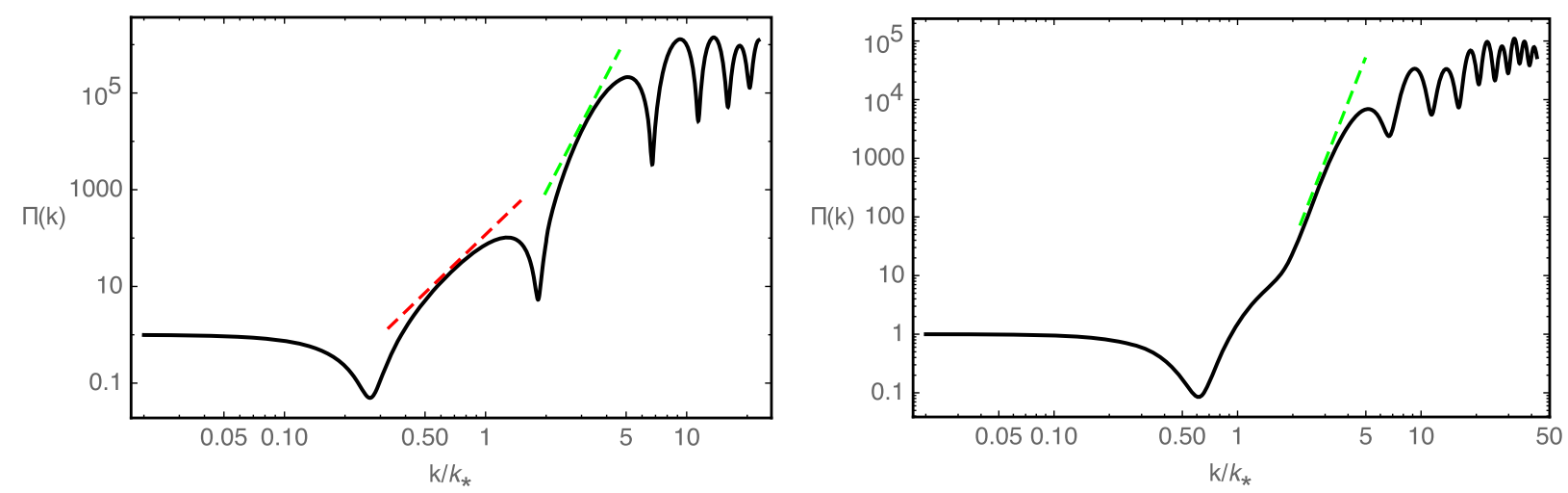

FIG. 7. Two examples of spectra computed with our analytic formulas. The green dashed line has a profile $k^{8}$, suggesting that the example of this section can in principle enhance the spectrum. The red dashed line has a profile $k^{4}$. Left: We have chosen the parameters $\alpha=250, \beta=200$ and $\Delta \tau_{A}=0.3, \Delta \tau_{B}=2.0, \Delta \tau_{C}=0.3, \Delta \tau_{D}=0.7$. Right: $\alpha=30, \beta=1000$ and $\Delta \tau_{A}=0.3, \Delta \tau_{B}=3.5, \Delta \tau_{C}=0.3$, $\Delta \tau_{D}=1.4$.

$\Pi_{\max }$, but it also depends on the duration of the nonattractor epochs as well on the intermediate de Sitter phase.

\section{The slope of the spectrum toward small scales}

An analysis of the slope of the spectrum is particularly interesting. As mentioned above, the spectral index can turn out to be larger in the phase of rapid growth of the spectrum, after the second intermediate dip occurs. Figure 7 represents the slope of the spectrum in two situations.

The green dashed curves have $n_{s}-1=8$, suggesting that this can be the maximal slope of the spectrum toward small scales, in the region of more enhanced growth. This overcomes considerably the $n_{s}-1=4$ limit of [10], that we also found in the previous section focusing on a single phase of non-slow-roll expansion.

This behavior can be analytically understood by means of our formulas, exploiting arguments we introduced in Sec. III A 2 for studying the slope of the spectrum. We compute the spectral index associated with the spectrum in this system, and focus on the limit of large $\alpha, \beta$ in a range of scales well after the first dip. Formulas are complex, but making appropriate expansions in regimes of small parameters they simplify considerably and are amenable of analytical investigation. First, we do an expansion of $n_{s}-1$ for small values of $\epsilon_{A}$ and $\epsilon_{C}$ and retain only the leading terms in the expansion, since we are interested to the limit of short duration of non-slow-roll epochs. We are left with the parameters $\epsilon_{B}, \epsilon_{D}$, and $k / k_{\star}$. From Fig. 6, we know that having a sizeable $\epsilon_{B}$ is essential for making manifest the enhanced slope of the spectrum at small scales. Instead, having a small $\epsilon_{D}$ allows for further amplify the spectrum at small scales: we can then use $\epsilon_{D}$ as additional small quantity for our arguments.

Namely, we make two different expansions of the spectral index in the two independent quantities $k / k_{\star}$ and $\epsilon_{D}$. In the first expansion we impose the hierarchy $k^{2} / k_{\star}^{2}<\epsilon_{D}$, which corresponds to relatively large scales between the two dips in the left panel of Fig 7. The spectral index results

$$
n_{s}-1=4-\frac{2 \epsilon_{B}^{3} k^{2}}{\epsilon_{D} k_{\star}^{2}}+\ldots
$$

where dots indicate subleading contributions in this regime. This formula matches with the red dashed line in the left panel of Fig 7. In the second expansion we consider smaller scales after the second dip, and impose the hierarchy $k^{2} / k_{\star}^{2}>\epsilon_{D}$. We then find

$$
n_{s}-1=8-\frac{2 \epsilon_{B} k^{2}}{5 k_{\star}^{2}}+\frac{8 \epsilon_{D} k_{\star}^{2}}{\epsilon_{B}^{3} k^{2}}+\ldots
$$

in agreement with the green dashed line in the left plot of Fig 7. These findings suggest that a succession of nonslow-roll phases in single-field inflation can much enhance the slope of the spectrum in single field inflation. Of course, we might expect that including three or more distinct separate phases of non-slow-roll evolution one can enhance the slope even more-we leave to future work an analysis of this fact and the consequences of these findings.

We conclude briefly discussing the possibility that the enhanced slope found here refers to the spectrum of curvature fluctuations, that being amplified can generate at second order a stochastic gravitational wave (GW) background. The scale-dependent profile of the latter might have an amplitude to be sufficiently large to be directly detectable with GW experiments. The formalism to study this process was developed in [45-47], and recently reconsidered or extended in various works (see, for example, [48,57,58]), also in view of applications to GW detectors. The work [59] provides relatively simple semianalytic formulas for the spectrum $\mathcal{P}^{(h)}$ of the $\mathrm{GW}$ induced at second-order from a scalar spectrum $\mathcal{P}^{(\zeta)} \cdot \mathcal{P}^{(h)}$ is written in terms of convolutions: 
$\mathcal{P}_{k}^{(h)}(\tau)=\int_{0}^{\infty} d v \int_{|1-v|}^{1+v} d u \mathcal{K}(\tau, u, v) \mathcal{P}_{u k}^{(\zeta)}(\tau) \mathcal{P}_{v k}^{(\zeta)}(\tau)$

The expression for the kernel $\mathcal{K}(\tau, u, v)$ is found in [59]. The associated energy density in GW as function of the scale is given by (the bar indicates time-averaging over oscillations)

$$
\Omega_{\mathrm{GW}}(\tau, k)=\frac{1}{24}\left(\frac{k}{a H}\right)^{2} \overline{\mathcal{P}}_{k}^{(h)}(\tau) .
$$

As representative examples, we consider three cases of curvature spectrum:

$$
\begin{aligned}
& \mathcal{P}_{k}^{(\zeta)}=\mathcal{A}_{s} \delta\left(\ln k / k_{\star}\right), \\
& \mathcal{P}_{k}^{(\zeta)}=4 \mathcal{A}_{s}\left(k / k_{\star}\right)^{4}, \\
& \mathcal{P}_{k}^{(\zeta)}=8 \mathcal{A}_{s}\left(k / k_{\star}\right)^{8},
\end{aligned}
$$

so to compare with the results of $[10,46]$. The normalizations have been chosen so to ensure that $\int d \ln k \mathcal{P}_{k}^{(\zeta)}=\mathcal{A}_{s}$ in all three cases. Power spectrum (3.32) corresponds to a $\delta$-like spectrum in log-space, peaked at a characteristic frequency $k_{\star}$; the power spectra (3.33) and (3.34) correspond respectively to power-laws with the maximal slope $n_{s}-1=4$ found in [10], and the maximal slope $n_{s}-1=8$ discussed above. Figure 8 represents the corresponding profiles of $\Omega_{\mathrm{GW}}(\tau, k)$ in each of the three cases.

We notice that the profile of the GW spectra in red and green are very similar, with the green one-corresponding to a profile for curvature perturbations spectrum scaling as $k^{8}$-slightly more amplified, and with more enhanced dip at intermediate scales. It would be interesting to study whether future GW experiments, for example using binning

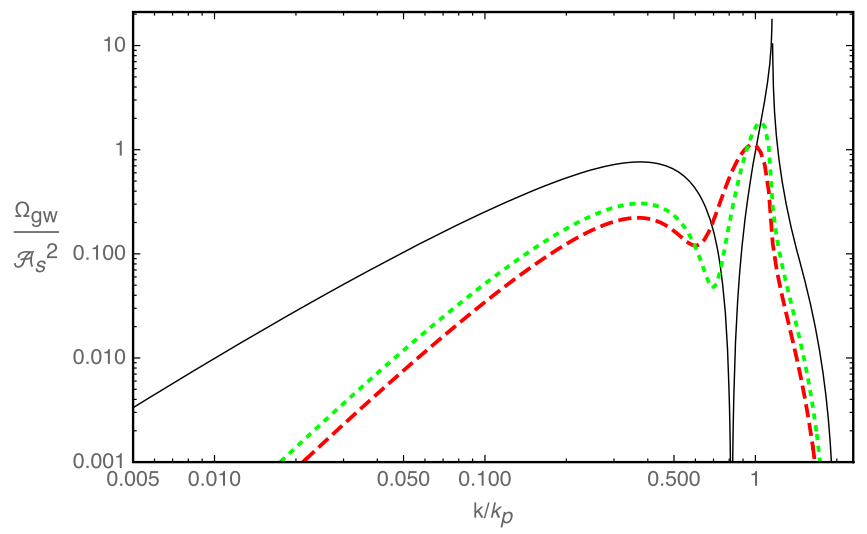

FIG. 8. Profile of the GW energy density $\Omega_{\mathrm{GW}}$ as a function of the scale. Continuous black line: $\delta$-like spectrum (3.32). Red dashed line: $k^{4}$-spectrum (3.33). Green dotted line: $k^{8}$-spectrum (3.34). techniques as proposed [60], can distinguish among these frequency profiles of GW spectra, in case of a detection of stochastic GW background.

\section{CONCLUSIONS}

We developed an analytical, model independent approach to study the spectrum of cosmological fluctuations (scalar or tensor) in single-field models of inflation that include brief epochs of violation of slow-roll conditions. Our analysis did not make any hypothesis on the value of the slow-roll parameters-instead we implemented a gradient expansion for solving the equations of fluctuations. We solved exactly these equations at leading order in a small parameter that controls the duration of non-slowroll evolution. Our approach leads to simple expressions for the fluctuation mode functions, depending on few parameters related with the slope of the pump field $z(\tau)$ during the phases of non-slow-roll evolution, and with the duration of the non-slow-roll eras. These parameters control the total enhancement and the slope of the fluctuation spectrum from large toward small scales. Our formulas for the power spectrum lead to qualitative features that are in overall agreement with what found in other numerical and analytical studies of concrete inflationary models with nonslow-roll phases, as the presence of dips, and of phases of rapid growth in the spectrum. This fact suggests that those features can be well described by our approximations of very short duration of non-slow-roll phase. As a byproduct of our methods, we first proposed new general relations connecting the position and depth of the more pronounced dip, with the maximal size of enhancement of the spectrum. Then, we confirmed that in the case of a single short phase of non-slow-roll evolution the steepest growth of the spectrum has spectral index $n_{s}-1=4$; on the other hand, if multiple non-slow-roll phases occur then this value can increase. In an example with two non-slow-roll phases, the maximal spectral index is $n_{s}-1=8$, indicating that the slope of the spectrum has memory of the total history of nonattractor phases during inflation.

These findings can be relevant when testing against observations the predictions of single-field models of inflation that include short non-slow-roll phases. The position of the most pronounced dip in the spectrum of fluctuations indicates the range of scales where predictions start to strongly deviate from slow-roll models. Knowledge of the dip properties can then be important when testing these scenarios against constraints on the large scale spectra obtained from CMB temperature fluctuations and spectral distortions. On the other hand, knowledge of the steepest growth of the spectrum is essential for understanding at what extent non-slow-roll single-field models are able to produce primordial black holes, or secondary stochastic gravitational wave spectra testable with current or future observations. 
Our method is based on a truncation of a Taylor series in the duration of non-slow-roll era at the first nonvanishing contribution: it is apparently better suited to study systems where slow-roll violation are sudden and last briefly. On the other hand, the resulting profiles of the fluctuation spectrum are consistent with previous literature in which the non-slow-roll phases can be more prolonged. It would be very interesting to understand more deeply the relations between different methods and findings. Moreover, in this work we focused on single-field inflation: it would be interesting to apply our methods also to models of multiple field inflation with slow-roll violation epochs, recently considered in $[61,62]$ using other approaches.

\section{ACKNOWLEDGMENTS}

It is a pleasure to thank Ogan Özsoy and Ivonne Zavala for useful discussions and their careful reading of the manuscript. G. T. is partially funded by STFC Grant No. ST/P00055X/1.

\section{APPENDIX A: AN EXAMPLE WITH ARBITRARILY FAST DECREASING PUMP FIELD}

In this Appendix, we identify the mode function $\psi_{k}$ with the curvature perturbation $\zeta_{k}$ in single field inflation with canonical kinetic terms. For simplicity, we work here with coordinate time $t$ related to conformal time by $d \tau \equiv$ $d t / a(t)$. Calling $\phi$ the homogeneous profile the scalar inflaton, the pump field reads

$$
z(t)=a(t) \frac{\dot{\phi}(t)}{H(t)} .
$$

If $\dot{\phi}$ and $H$ are nearly constant as in single field slow-roll inflation, then the pump field $z \propto a$; so it increases with time during inflation [in conformal time, its profile is well approximated by Eq. (2.3)]. On the other hand, models exist where the scalar velocity $\dot{\phi}$ transiently decrease very fast, even in a regime of nearly de Sitter expansion. In those cases, the pump field decreases with time, and we enter in a brief regime of nonattractor.

In this Appendix we build a representative single-field model to demonstrate that the decreasing rate can be arbitrarily large. Working in nearly de Sitter space, we consider a system where during a short epoch the scalar derivative decreases arbitrarily fast. We build the model following the approach of Starobinsky [31], who considered cases of a linear potential $V(\phi)$ that is continuous, but with a sudden change of slope at a certain value $\bar{\phi}$ of the scalar. In such a system, for a short time interval, the scalar velocity $\dot{\phi}$ decreases as the cube of the scale factor, $1 / a^{3}$, and during such interval the pump field decreases with time.
We now show that, by selecting an appropriate setup, the scalar velocity can decrease during a short nonattractor phase as $\dot{\phi} \propto 1 / a^{\beta}$ for arbitrary $\beta$. This dimensionless parameter $\beta$ controls the rate of variation of the pump field. For simplicity, to present our system it is convenient to adopt a Hamilton-Jacobi approach $[63,64]$, in which the Hubble parameter is expressed as function of the scalar: $H=H(\phi)$. Setting $M_{\mathrm{Pl}}=1$, the system of homogeneous equations reads

$$
\begin{gathered}
\dot{\phi}=-2 H_{, \phi}(\phi), \\
H^{2}(\phi)=\frac{V(\phi)}{3}+\frac{2 H_{, \phi}(\phi)^{2}}{3},
\end{gathered}
$$

with $V(\phi)$ the scalar potential. It is not hard to show that the results of this method are equivalent to the more traditional approach based on Lagrange equations, in the limit of homogeneous evolution (see [63]).

Inspired by the Starobinsky model [31], we choose a profile for the Hubble parameter that is continuous but has a jump in its first derivative at $\phi=\bar{\phi}$. It is

$$
\begin{aligned}
H(\phi)= & H_{0}+\frac{H_{1}}{2} \phi+\frac{H_{1} H_{2}^{( \pm)}}{2 \beta H_{0}} e^{-\beta_{H_{1}}^{H_{0}} \phi} \\
& +\Theta[\phi-\bar{\phi}]\left(\frac{H_{2}^{(-)}-H_{2}^{(+)}}{2 \beta H_{0}} H_{1} e^{-\beta_{H_{1}}^{H_{0}} \bar{\phi}}\right),
\end{aligned}
$$

where $H_{0}, H_{1}, H_{2}, \beta$ are arbitrary constants. The suffixes $( \pm)$ in $\mathrm{H}_{2}$ indicate that this quantity is computed on the right or on the left of $\bar{\phi}$. The constant quantity $H_{0}$ shall represent the nearly constant value of the Hubble parameter during inflation, that receives small correction by the remaining terms (we assume $H_{1,2} \ll H_{0}$ ). In such case, during all stages $a(t)=e^{H_{0} t}$ at good approximation. The last term in expression (A4) is proportional to a Heaviside $\Theta$-function and makes the Hubble parameter continuous at the position $\phi=\bar{\phi}$. On the other hand, the potential $V(\phi)$, which can be read from Eq. (A3), has discontinuities in $\phi$.

It is straightforward to solve Eq. (A2). The scalar field velocity results

$$
\dot{\phi}(t)=-\frac{H_{1}^{2} \phi_{0} e^{-\beta H_{0} t}}{H_{2}^{( \pm)}+H_{1} \phi_{0} e^{-\beta H_{0} t}},
$$

with $\phi_{0}$ an arbitrary constant. We start with values $\phi>\bar{\phi}$, and we assume that in such stage $H_{2}^{(+)} \ll H_{1} \phi_{0} e^{-\beta H_{0} t}$. Then, in this phase $\dot{\phi} \simeq-H_{1}$, and the pump field (A1) increases with time as in single-field inflation.

Then, at $\phi=\bar{\phi}$, we enter in the second region where $H_{2}^{(-)} \gg H_{1} \phi_{0} e^{-\beta H_{0} t}$. In such case, the scalar velocity decreases very rapidly 


$$
\dot{\phi} \simeq-\frac{H_{1}^{2} \phi_{0} e^{-\beta H_{0} t}}{H_{2}^{(-)}} \Rightarrow \dot{\phi} \propto \frac{1}{a^{\beta}}
$$

hence, since $\beta$ is a free parameter, the pump field (A1) can decrease as rapidly as we desire. This nonattractor phase can then end by adding yet another contribution to the Hubble parameter to slow-down again the scalar velocity. This concrete example shows that there is in principle no bound on the rate of decrease of the pump field in single field inflation.

\section{APPENDIX B: SPECTRAL INDEX AND MULTIPLE NON-SLOW-ROLL EPOCHS}

In Sec. III B we found that for inflationary processes containing two eras of non-slow-roll evolution, separated by an epoch of standard slow-roll expansion, the slope of the spectrum can increase from $n_{S}-1=4$ to $n_{s}-1=8$ well after the first dip. Here we study the same phenomenon using two alternative approaches, to qualitatively confirm our findings. We focus on the dynamics of the curvature perturbation $\zeta_{k}$ in single-field inflation, for which the pump field is given by (A1).

\section{Israel junction conditions}

The first approach we use is the analytical method of Israel junction conditions between phases of constant slowroll parameters, as used in [10].

We study the simplest possible situation, where two phases of ultra-slow-roll evolution characterized by slowroll parameters $\epsilon_{1} \simeq 0$ and $\epsilon_{2} \simeq-6$ [see the definition around Eq. (2.6)] contain in between a phase of quasi-de Sitter expansion (where for simplicity, we set $\epsilon_{1} \simeq \epsilon_{2}=0$ ). The system is pictorially depicted in Fig. 5, where the green regions are the slow-roll, the blue regions the ultra-slowroll phases.

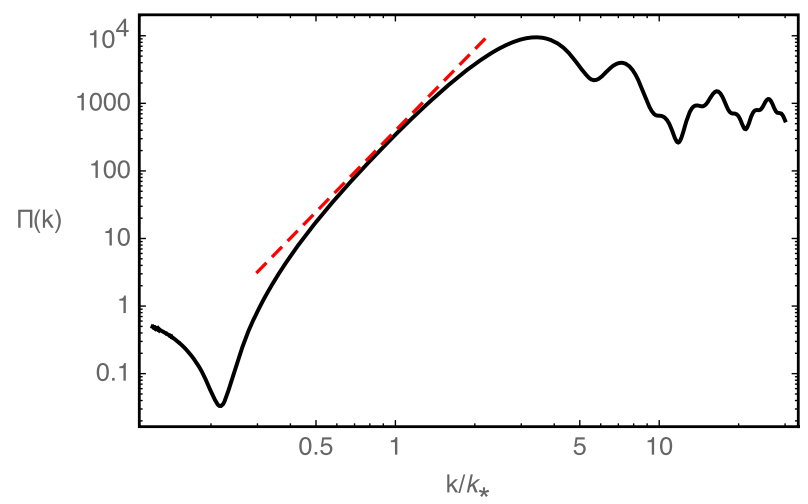

The general mode functions during a pure de Sitter evolution are given in Eq. (2.4). During ultra-slow-roll, a straightforward computation (or the duality arguments of [23]) gives the following expression for the mode function $\psi_{k}^{\mathrm{USR}}(\tau)$ solving the evolution equations

$$
\psi_{k}^{\mathrm{USR}}(\tau)=\left(\frac{\bar{\tau}}{\tau}\right)^{3} \psi_{k}^{\mathrm{dS}}(\tau)
$$

where $\bar{\tau}$ is a reference time scale, and $\psi_{k}^{\mathrm{dS}}(\tau)$ the general solution (2.4) during the de Sitter epoch. Applying standard Israel matching conditions at the boundaries between phases as in [10], we get the expression of mode functions that allows one to compute the spectrum of superhorizon modes at the end of inflation.

Figure 9 (left panel) shows the resulting spectrum after a single phase of nonattractor. Within this approach based on Israel conditions the position of the dip and its relation to the maximal growth of the spectrum can be analytically computed. In fact, we already shown in the main text-see our discussion around Eq. (3.22), comparing our results with [10]-that relation (3.19), stating that $k / k_{\star} \propto \sqrt{3 / 2} \Pi_{\max }^{-1 / 4}$, is obeyed fairly well.

Figure 9 (right panel) contains an example of spectrum evaluated following these methods, which clearly shows that an initial grow with (at most) $n_{S}-1=4$ spectral tilt is followed by a second phase with where the spectral tilt can reach $n_{S}-1=8$.

\section{Numerical method}

We can investigate this phenomenon using numerical methods applied to a simple generalization of Starobinsky model [31].

The model of [31] (see also [37], the discussion in their Sec. IV) consists of a linear inflationary potential $V(\phi)$ that is continuous, but with a jump in its first derivative at a given value of the scalar $\phi$. We can generalize the potential

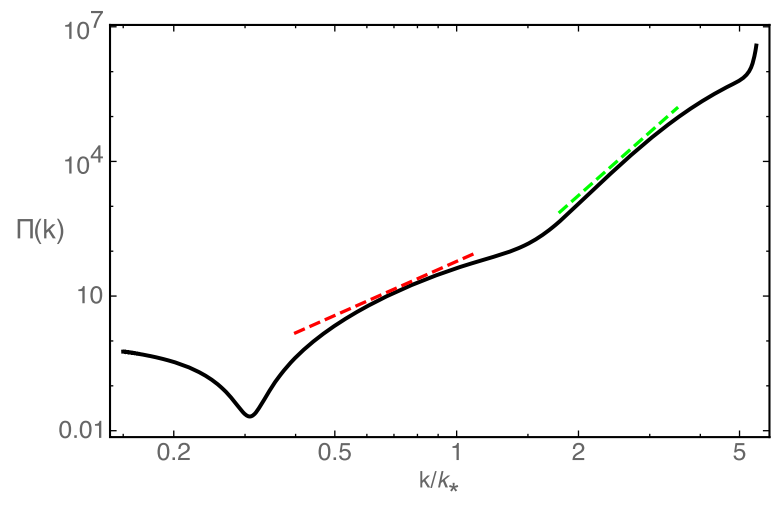

FIG. 9. Spectrum of super-horizon fluctuations evaluated at the end of inflation for an inflationary system containing one (left panel) or two (right panel) phases of ultra-slow-roll inflation. This plot was produced using a method based on Israel junction conditions, as [10]. Red dashed lines have slope $k^{4}$, green dashed lines have slope $k^{8}$. Left: a single-phase of nonattractor. Right: two phases of nonattractor, lasting respectively 0.2 e-fold and 1.7 e-fold, containing a 1.3 e-fold epoch of normal slow-roll in between. 

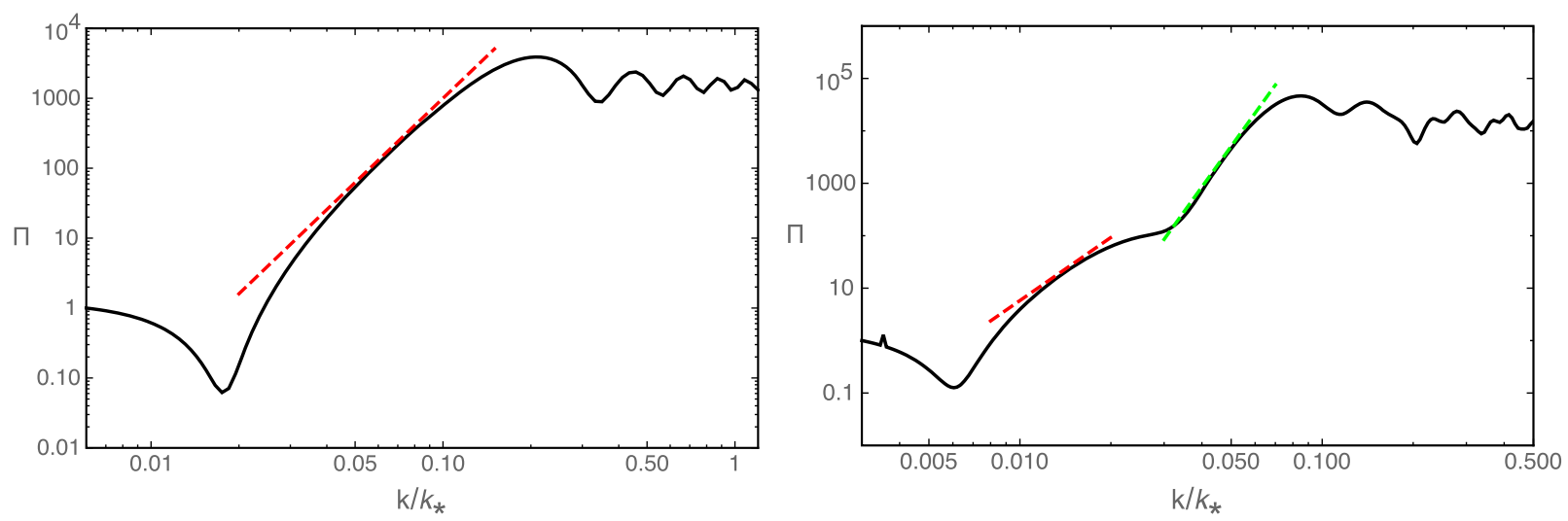

FIG. 10. Spectrum of superhorizon fluctuations evaluated at the end of inflation for an inflationary system containing one (left panel), or two (right panel) phases of nonattractor evolution. This plot was produced starting from the generalization (B2) of the Starobinsky model [31,37]. Red dashed lines have slope $k^{4}$, green dashed lines have slope $k^{8}$. Left: a single-phase of nonattractor. Right: two phases of nonattractor with a nested epoch of standard slow-roll expansion. The first nonattractor phase lasts 1.4 e-fold, and the duration of slow-roll expansion is 2 e-folds. We choose the parameters $A_{1}=90, A_{2}=15, A_{4}=3$, while $A_{3}$ satisfies conditions to ensure continuity of the scalar first derivative.

allowing for several discontinuities in the first derivative of the potential (while the potential itself is continuous):

$$
V(\phi)= \begin{cases}V_{0}+A_{1}\left(\phi-\phi_{1}\right) & \text { for } \phi>\phi_{1}, \\ V_{0}+A_{2}\left(\phi-\phi_{1}\right) & \text { for } \phi_{2} \leq \phi \leq \phi_{1}, \\ V_{0}+A_{3}\left(\phi-\phi_{2}\right) & \text { for } \phi_{3} \leq \phi \leq \phi_{2}, \\ V_{0}+A_{4}\left(\phi-\phi_{3}\right) & \text { for } \phi<\phi_{3},\end{cases}
$$

where $V_{0}, A_{i}, \phi_{i}$ are constant parameters. We choose $V_{0}$ large enough to ensure that the scale factor can be well approximated by de Sitter space with a nearly constant Hubble parameter $H_{0}$ and $a(t) \simeq e^{H_{0} t}$. It is simple to solve the evolution equation for the scalar field $\phi$ in each of the ranges appearing in Eq. (B2). The structure of the solution is

$$
3 H_{0} \dot{\phi}(t)=-A_{i}+c_{i} / a^{3}(t)
$$

where the $A_{i}$ are the parameters appearing in Eq. (B2), while the $c_{i}$ are integration constants chosen to ensure that the first derivative is continuous at the transition points $\phi_{i}$. As shown in $[31,37]$, if there is a large hierarchy between the quantities $A_{i}$, one can design situations where periods of slow-roll evolution with $3 H_{0} \dot{\phi} \simeq$ constant are nested between periods of nonattractor phases with $3 H_{0} \dot{\phi} \propto 1 / a^{3}(t)$. Figure 10 contains two examples of numerical evaluation of the curvature fluctuation spectrum for this system.

In the left we plot the original Starobinsky example $[31,37]$, with a single discontinuity in the first derivative of the potential, leading to a single phase of nonattractor evolution: the spectrum of curvature fluctuations grow with a maximal slope $k^{4}$. In the original example the single nonattractor phase is relatively prolonged, in order to sufficiently increase the spectrum. Hence we can not expect that our formula (3.19) relating dip position with the spectrum maximal enhancement is too accurate, since we derived Eq. (3.19) in the limit of short nonattractor phase. On the other hand, we numerically evaluated the ratio of the dip position versus the scale of nonattractor inception for our example, finding the value 0.27. By computing the value of maximal enhancement of the spectrum we find the relation $\sqrt{3 / 2} \Pi_{\max }^{-1 / 4}=0.15$. Hence, Eq. (3.19) is satisfied up to a factor of order 2 .

In the right plot of Fig. 10 we instead use the complete potential (B2), and we study two phases of nonattractor, with a nested epoch of slow-roll in between. We notice that toward small scales the spectrum increases its slope reaching $n_{S}-1=8$, as desired.

These preliminary findings support the results of the main text. It would be interesting to carefully study the system in a more realistic setup. We defer this question to future work. 
[1] V. Mukhanov, Physical Foundations of Cosmology (Cambridge University Press, Oxford, 2005).

[2] J. Garcia-Bellido and E. Ruiz Morales, Primordial black holes from single field models of inflation, Phys. Dark Universe 18, 47 (2017).

[3] J. M. Ezquiaga, J. Garcia-Bellido, and E. Ruiz Morales, Primordial black hole production in critical Higgs inflation, Phys. Lett. B 776, 345 (2018).

[4] G. Ballesteros and M. Taoso, Primordial black hole dark matter from single field inflation, Phys. Rev. D 97, 023501 (2018).

[5] M. P. Hertzberg and M. Yamada, Primordial black holes from polynomial potentials in single field inflation, Phys. Rev. D 97, 083509 (2018).

[6] H. Motohashi and W. Hu, Primordial black holes and slowroll violation, Phys. Rev. D 96, 063503 (2017).

[7] B. Carr, F. Kuhnel, and M. Sandstad, Primordial black holes as dark matter, Phys. Rev. D 94, 083504 (2016).

[8] M. Sasaki, T. Suyama, T. Tanaka, and S. Yokoyama, Primordial black holes perspectives in gravitational wave astronomy, Classical Quantum Gravity 35, 063001 (2018).

[9] N. Bartolo et al., Science with the space-based interferometer LISA. IV: Probing inflation with gravitational waves, J. Cosmol. Astropart. Phys. 12 (2016) 026.

[10] C. T. Byrnes, P. S. Cole, and S. P. Patil, Steepest growth of the power spectrum and primordial black holes, J. Cosmol. Astropart. Phys. 06 (2019) 028.

[11] P. Carrilho, K. A. Malik, and D. J. Mulryne, Dissecting the growth of the power spectrum for primordial black holes, Phys. Rev. D 100, 103529 (2019).

[12] O. Ozsoy and G. Tasinato, On the slope of curvature power spectrum in non-attractor inflation, J. Cosmol. Astropart. Phys. 04 (2020) 048.

[13] J. Chluba, J. Hamann, and S. P. Patil, Features and new physical scales in primordial observables: Theory and observation, Int. J. Mod. Phys. D 24, 1530023 (2015).

[14] W. H. Kinney, Horizon crossing and inflation with large eta, Phys. Rev. D 72, 023515 (2005).

[15] C. Germani and T. Prokopec, On primordial black holes from an inflection point, Phys. Dark Universe 18, 6 (2017).

[16] K. Dimopoulos, Ultra slow-roll inflation demystified, Phys. Lett. B 775, 262 (2017).

[17] Z. Yi and Y. Gong, On the constant-roll inflation, J. Cosmol. Astropart. Phys. 03 (2018) 052.

[18] C. Pattison, V. Vennin, H. Assadullahi, and D. Wands, The attractive behaviour of ultra-slow-roll inflation, J. Cosmol. Astropart. Phys. 08 (2018) 048.

[19] S. Inoue and J. Yokoyama, Curvature perturbation at the local extremum of the inflaton's potential, Phys. Lett. B 524, 15 (2002).

[20] A. D. Linde, Fast roll inflation, J. High Energy Phys. 11 (2001) 052.

[21] J. Martin, H. Motohashi, and T. Suyama, Ultra slow-roll inflation and the non-Gaussianity consistency relation, Phys. Rev. D 87, 023514 (2013).

[22] H. Motohashi, A. A. Starobinsky, and J. Yokoyama, Inflation with a constant rate of roll, J. Cosmol. Astropart. Phys. 09 (2015) 018.

[23] D. Wands, Duality invariance of cosmological perturbation spectra, Phys. Rev. D 60, 023507 (1999).
[24] M. Mylova, O. zsoy, S. Parameswaran, G. Tasinato, and I. Zavala, A new mechanism to enhance primordial tensor fluctuations in single field inflation, J. Cosmol. Astropart. Phys. 12 (2018) 024.

[25] O. Ozsoy, M. Mylova, S. Parameswaran, C. Powell, G. Tasinato, and I. Zavala, Squeezed tensor non-Gaussianity in non-attractor inflation, J. Cosmol. Astropart. Phys. 09 (2019) 036.

[26] D. Roberts, A. R. Liddle, and D. H. Lyth, False vacuum inflation with a quartic potential, Phys. Rev. D 51, 4122 (1995).

[27] O. Seto, J. Yokoyama, and H. Kodama, What happens when the inflaton stops during inflation, Phys. Rev. D 61, 103504 (2000).

[28] S. M. Leach and A. R. Liddle, Inflationary perturbations near horizon crossing, Phys. Rev. D 63, 043508 (2001).

[29] R. K. Jain, P. Chingangbam, and L. Sriramkumar, On the evolution of tachyonic perturbations at super-Hubble scales, J. Cosmol. Astropart. Phys. 10 (2007) 003.

[30] R. K. Jain, P. Chingangbam, J.-O. Gong, L. Sriramkumar, and T. Souradeep, Punctuated inflation and the low CMB multipoles, J. Cosmol. Astropart. Phys. 01 (2009) 009.

[31] A. A. Starobinsky, Spectrum of adiabatic perturbations in the universe when there are singularities in the inflation potential, Pis'ma Zh. Eksp. Teor. Fiz. 55, 477 (1992) [JETP Lett. 55, 489 (1992)].

[32] J. A. Adams, G. G. Ross, and S. Sarkar, Multiple inflation, Nucl. Phys. B503, 405 (1997).

[33] J. A. Adams, B. Cresswell, and R. Easther, Inflationary perturbations from a potential with a step, Phys. Rev. D 64, 123514 (2001).

[34] M. Biagetti, G. Franciolini, A. Kehagias, and A. Riotto, Primordial black holes from inflation and quantum diffusion, J. Cosmol. Astropart. Phys. 07 (2018) 032.

[35] J. M. Ezquiaga and J. Garca-Bellido, Quantum diffusion beyond slow-roll: Implications for primordial black-hole production, J. Cosmol. Astropart. Phys. 08 (2018) 018.

[36] H. Firouzjahi, A. Nassiri-Rad, and M. Noorbala, Stochastic ultra slow roll inflation, J. Cosmol. Astropart. Phys. 01 (2019) 040 .

[37] S. M. Leach, M. Sasaki, D. Wands, and A. R. Liddle, Enhancement of superhorizon scale inflationary curvature perturbations, Phys. Rev. D 64, 023512 (2001).

[38] M. Cicoli, V. A. Diaz, and F. G. Pedro, Primordial black holes from string inflation, J. Cosmol. Astropart. Phys. 06 (2018) 034.

[39] O. Ozsoy, S. Parameswaran, G. Tasinato, and I. Zavala, Mechanisms for primordial black hole production in string theory, J. Cosmol. Astropart. Phys. 07 (2018) 005.

[40] E. Bugaev and P. Klimai, Constraints on the induced gravitational wave background from primordial black holes, Phys. Rev. D 83, 083521 (2011).

[41] J. Chluba, A. L. Erickcek, and I. Ben-Dayan, Probing the inflaton: Small-scale power spectrum constraints from measurements of the CMB energy spectrum, Astrophys. J. 758, 76 (2012).

[42] T. Nakama, B. Carr, and J. Silk, Limits on primordial black holes from $\mu$ distortions in cosmic microwave background, Phys. Rev. D 97, 043525 (2018). 
[43] G. Sato-Polito, E. D. Kovetz, and M. Kamionkowski, Constraints on the primordial curvature power spectrum from primordial black holes, Phys. Rev. D 100, 063521 (2019).

[44] C. Unal, E. D. Kovetz, and S. P. Patil, Multi-messenger probes of massive black holes from enhanced primordial fluctuations, arXiv:2008.11184.

[45] S. Mollerach, D. Harari, and S. Matarrese, CMB polarization from secondary vector and tensor modes, Phys. Rev. D 69, 063002 (2004).

[46] K. N. Ananda, C. Clarkson, and D. Wands, The cosmological gravitational wave background from primordial density perturbations, Phys. Rev. D 75, 123518 (2007).

[47] D. Baumann, P. J. Steinhardt, K. Takahashi, and K. Ichiki, Gravitational wave spectrum induced by primordial scalar perturbations, Phys. Rev. D 76, 084019 (2007).

[48] R. Saito and J. Yokoyama, Gravitational Wave Background as a Probe of the Primordial Black Hole Abundance, Phys. Rev. Lett. 102, 161101 (2009); , Erratum, Phys. Rev. Lett. 107, 069901 (2011).

[49] L. Lentati et al., European pulsar timing array limits on an isotropic stochastic gravitational-wave background, Mon. Not. R. Astron. Soc. 453, 2577 (2015).

[50] Z. Arzoumanian et al. (NANOGrav Collaboration), The NANOGrav 12.5-year data set: Search for an isotropic stochastic gravitational-wave background, arXiv:2009.04496.

[51] S. Parameswaran, G. Tasinato, and I. Zavala, Subleading effects and the field range in axion inflation, J. Cosmol. Astropart. Phys. 04 (2016) 008.

[52] N. C. Bizet, O. Loaiza-Brito, and I. Zavala, Mirror quintic vacua: Hierarchies and inflation, J. High Energy Phys. 10 (2016) 082.

[53] T. Kobayashi, S. Uemura, and J. Yamamoto, Polyinstanton axion inflation, Phys. Rev. D 96, 026007 (2017).
[54] O. Ozsoy, Gravitational waves from a rolling axion monodromy, arXiv:2005.10280.

[55] E. Silverstein and A. Westphal, Monodromy in the CMB: Gravity waves and string inflation, Phys. Rev. D 78, 106003 (2008).

[56] L. McAllister, E. Silverstein, and A. Westphal, Gravity waves and linear inflation from axion monodromy, Phys. Rev. D 82, 046003 (2010).

[57] T. Nakama and T. Suyama, Primordial black holes as a novel probe of primordial gravitational waves. II: Detailed analysis, Phys. Rev. D 94, 043507 (2016).

[58] J. Garcia-Bellido, M. Peloso, and C. Unal, Gravitational waves at interferometer scales and primordial black holes in axion inflation, J. Cosmol. Astropart. Phys. 12 (2016) 031.

[59] K. Kohri and T. Terada, Semianalytic calculation of gravitational wave spectrum nonlinearly induced from primordial curvature perturbations, Phys. Rev. D 97, 123532 (2018).

[60] C. Caprini, D. G. Figueroa, R. Flauger, G. Nardini, M. Peloso, M. Pieroni, A. Ricciardone, and G. Tasinato, Reconstructing the spectral shape of a stochastic gravitational wave background with LISA, J. Cosmol. Astropart. Phys. 11 (2019) 017.

[61] G. A. Palma, S. Sypsas, and C. Zenteno, Seeding Primordial Black Holes in Multifield Inflation, Phys. Rev. Lett. 125, 121301 (2020).

[62] J. Fumagalli, S. Renaux-Petel, J. W. Ronayne, and L. T. Witkowski, Turning in the landscape: A new mechanism for generating primordial black holes, arXiv:2004.08369.

[63] D. S. Salopek and J. R. Bond, Nonlinear evolution of long wavelength metric fluctuations in inflationary models, Phys. Rev. D 42, 3936 (1990).

[64] C. T. Byrnes and G. Tasinato, Non-Gaussianity beyond slow roll in multi-field inflation, J. Cosmol. Astropart. Phys. 08 (2009) 016. 